\title{
Electroacupuncture at GV20-GB7 regulates mitophagy to protect against neurological deficits following intracerebral hemorrhage via inhibition of apoptosis
}

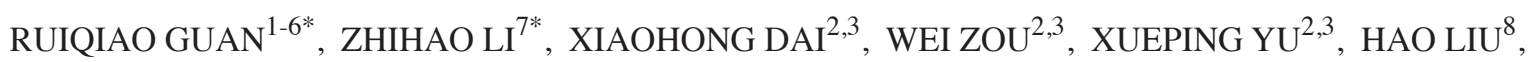

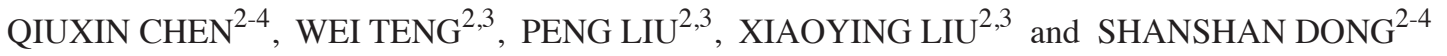 \\ ${ }^{1}$ Department of Integrated Chinese and Western Medicine, Zhongshan Hospital, Fudan University, Shanghai 200032; \\ ${ }^{2}$ Department of Clinical Medicine, Heilongjiang University of Chinese Medicine; ${ }^{3}$ The Third Department of Acupuncture \\ and Moxibustion, First Affiliated Hospital of Heilongjiang University of Chinese Medicine; ${ }^{4}$ Clinical Key Laboratory of \\ Integrated Chinese and Western Medicine, Heilongjiang University of Chinese Medicine, Harbin, Heilongjiang 150040, \\ P.R. China; ${ }^{5}$ Department of Traditional Chinese Medicine, London South Bank University, London SE1 6RD; \\ ${ }^{6}$ The Clinic of Traditional Chinese Medicine, London Confucius Institute of Traditional Chinese Medicine, London SE1 0AA, UK; \\ ${ }^{7}$ Department of Acupuncture and Moxibustion, Yueyang Hospital of Integrated Traditional Chinese and Western Medicine, \\ Shanghai University of Chinese Medicine, Shanghai 200437; ${ }^{8}$ Department of Acupuncture and Moxibustion, \\ Tongde Hospital of Zhejiang Province, Hangzhou, Zhejiang 315099, P.R. China
}

Received July 23, 2020; Accepted February 24, 2021

DOI: $10.3892 / \mathrm{mmr} .2021 .12131$

\begin{abstract}
The acupuncture penetrating line of Baihui (GV20) to Qubin (GB7) spans the parietal, frontal and temporal lobes. The present study aimed to elucidate the mechanism by which electroacupuncture (EA) at GV20-GB7 regulates mitophagy in intracerebral hemorrhage (ICH) and whether it serves a neuroprotective role. A whole blood-induced ICH model was used. Mitophagy-regulating proteins, including BCL/adenovirus E1B $19 \mathrm{kDa}$-interacting protein 3 (BNIP3), PTEN-induced putative kinase 1 (PINK1), Parkin and apoptosis-associated proteins were detected by western blotting; autophagy following ICH was evaluated by immunofluorescent techniques; morphological characteristics of mitophagy were observed using transmission electron microscopy; and TUNEL assay was performed to determine the number of apoptotic cells. Immunohistochemistry was used to detect p53 expression. The protective role of EA (GV20-GB7) via enhanced mitophagy and suppressed apoptosis in ICH was further confirmed by decreased modified neurological severity score. The results showed that EA (GV20-GB7)
\end{abstract}

Correspondence to: Dr Wei Zou, The Third Department of Acupuncture and Moxibustion, First Affiliated Hospital of Heilongjiang University of Chinese Medicine, 26 Heping Road, Xiangfang, Harbin, Heilongjiang 150040, P.R. China

E-mail: visa4journals@163.com

*Contributed equally

Key words: intracerebral hemorrhage, electroacupuncture, autophagy, mitophagy, apoptosis treatment upregulated mitochondrial autophagy following $\mathrm{ICH}$ and inhibited apoptotic cell death. The mechanism underlying EA (GV20-GB7) treatment may involve inhibition of p53, an overlapping protein of autophagy and apoptosis. EA (GV20-GB7) treatment decreased neurobehavioral deficits following ICH but pretreatment with 3-methyladenine counteracted the beneficial effects of EA (GV20-GB7) treatment. In conclusion, EA (GV20-GB7) improved recovery from ICH by regulating the balance between mitophagy and apoptosis.

\section{Introduction}

Intracerebral hemorrhage $(\mathrm{ICH})$ is a stroke subtype with a high mortality rate, which often results in neurological impairments that can cause long-term disability (1). In the USA, the mortality rate is $35-52 \%$ within 30 days of $\mathrm{ICH}$, with half of deaths occurring in the first 2 days, and only $20 \%$ expectation of functional independence at 6 months among an estimated 67,000 patients $(2,3)$. Non-traumatic ICH is due to the rupture of blood vessels in the brain and subsequent tissue compression (4). The initial bleed causes hematoma mass that results in physical disruption of brain tissue; the pathophysiological processes of secondary injury following ICH are characterized by ischemia, edema, apoptosis and necrosis surrounding the hematoma $(5,6)$.

Autophagy is a process by which cells recycle cellular components, and degrade excess or defective organelles, thereby participating in cytoplasmic component turnover and protein quality control, which can prompt responses to nutrient depletion and pathological stress $(7,8)$. Autophagy is characterized by degradation of macromolecular intracellular material by double-membrane autophagic vesicles (known as autophagosomes) and also serves a constitutive role in the elimination 
of mitochondria (9). This specific regulated mechanism of selective mitochondria degradation via autophagy is defined as mitophagy (10). Mitophagy is involved in mitochondrial maintenance, cellular differentiation and cell survival in mammalian systems (11). An association between mitophagy and mitochondrial function maintenance may underly the pathology of certain diseases, aging, neurodegeneration and stroke (12). PTEN-induced putative kinase 1 (PINK1), Parkin, BCL/adenovirus E1B 19 kDa-interacting protein 3 (BNIP3), NIX/BNIP3L (BNIP3-like) and FUN14 domain containing 1 have been identified as mitophagy receptors in mammalian cells (13). Previous studies $(14,15)$ of stroke have demonstrated that enhanced mitophagy serves a key role in ameliorating secondary cell death and associated complications. A study involving traumatic brain injury (TBI) showed that enhancing mitophagy following TBI by melatonin treatment ameliorates neuronal death and behavioral deficit by negatively regulating inflammation activation and IL-1 $\beta$ secretion via autophagy of damaged mitochondria through the mTOR pathway (14). One study established that activated mitophagy may inhibit inflammasome-mediated cell death and decrease reactive oxygen species generation following induction of subarachnoid hemorrhage (16). Furthermore, mitophagy may contribute to the pro-survival apoptosis pathway by decreasing cytochrome $c$ release capacity (17). However, the question of whether mitophagy contributes to cell death in stroke remains controversial.

Electroacupuncture (EA) is a modern technique based on a combination of the meridian theory of Traditional Chinese Medicine and transcutaneous electrical stimulation therapy (18). Recommended as a complementary treatment for stroke, EA has shown promise in stroke rehabilitation (19). The benefits of EA are attributed to its readily quantifiable stimulation parameters of frequency, intensity and duration $(20,21)$. The technique has been applied to ICH models in a previous study (22) and has been reported to effectively decrease blood-brain barrier permeability and improve neurological recovery (23). Researchers have focused on the regulation of autophagy by EA treatment in stroke, showing that EA may affect ultrastructure and autophagy-associated factors $(24,25)$. For example, EA treatment was demonstrated to exert a neuroprotective effect following ischemic stroke by inhibiting autophagy via restricting autophagosome formation and mediating the mTORC1-unc-51-like autophagy activating kinase 1 complex-Beclin1 pathway (26). Other research has shown that EA (at GV20) pretreatment may exert a protective effect on cerebral ischemic and reperfusion injury by upregulating autophagy via enhanced expression of LC3 and downregulated phosphorylated-mTOR (27). The present study focused on acupoint GV20-GB7 (Baihui-Qubin). Our previous study (28) investigated the effectiveness of GV20-GB7 using an ICH rat model and found that acupuncture at GV20-GB7 may suppress neuronal differentiation of neural stem cells by inhibiting the Notch-Hes signaling pathway and maintaining neural stem cell proliferation. Furthermore, acupuncture at GV20-GB7 has been reported to improve the recovery of neurological function by decreasing the inflammatory response via inhibition of TNF- $\alpha / \mathrm{NF} \kappa \mathrm{B}$ expression levels (29). The present study aimed to elucidate the mechanism by which EA at GV20-GB7 regulates mitophagy in ICH and whether it serves a neuroprotective role. It was hypothesized that EA at GV20-GB7 could inhibit apoptotic cell death following $\mathrm{ICH}$ by regulating the mitophagy process, thereby alleviating symptoms of neurological impairment induced by $\mathrm{ICH}$.

\section{Materials and methods}

Experimental animals. Male Sprague-Dawley rats (weight, 300-350 g) were obtained from the Experimental Animal Center of Heilongjiang University of Chinese Medicine [license no. SYXK (Hei) 2017061001]. Animals were housed in controlled conditions under a $12-\mathrm{h}$ light/dark cycle, at $22 \pm 2^{\circ} \mathrm{C}$, $60-70 \%$ humidity and noise $<60 \mathrm{~dB}$, with ad libitum access to food and water. All experiments were approved by the Animal Care and Use Committee of Heilongjiang University of Chinese Medicine and performed in accordance with the National Institutes of Health Guide for the Care and Use of Laboratory Animals (30).

ICH model and groups. Rats were anesthetized with pentobarbital [60 mg/kg; intraperitoneal (i.p.) injection], then shaved at the scalp. Aseptic techniques were used for surgical procedures, as described in our previous study (29). Briefly, a $\sim 1-\mathrm{cm}$ midline incision was made, exposing a perpendicular intersection point of the coronal and sagittal suture. A $0.1-\mathrm{mm}$ hole was drilled in the skull $(0.2 \mathrm{~mm}$ anterior and $3.5 \mathrm{~mm}$ laterally to the right of bregma). Subsequently, $50 \mu 1$ autologous whole blood from the tail artery was injected into the basal ganglia via Hamilton syringe at $5 \mathrm{~mm}$ depth below the skull surface and the needle was left in place for $5 \mathrm{~min}$. The sham-operated group received intracerebral needle insertion only, without blood injection. The burr hole was sealed with dental zinc phosphate cement and the skin was sutured. Following surgery, each animal was placed in a clean cage with free access to food and water. The success rate of the autologous blood injection operation, which indicated ICH was successfully induced, was $~ 95 \%$. Rats in the control group of each time point did not undergo surgery.

Since the morphology of mitophagy has been observed in previous studies (31-34), the experiments were repeated at least three times to confirm mitophagy as a phenomenon in $\mathrm{ICH}$. A total of 5 rats/group were used for transmission electron microscopy (TEM), as previously described $(35,36)$. A total of 345 rats were used in the study. In order to determine the time-dependent effect of ICH on mitophagy, 120 rats were randomly and evenly divided into a control and $\mathrm{ICH}$ model group ( $n=60 /$ group). Tests were performed 6 and $24 \mathrm{~h}$, and 3 and 7 days after ICH. In order to evaluate the effect of EA on mitophagy following ICH, 225 rats were randomly assigned to five treatment groups, each of which comprised four experimental subgroups ( 6 and 24 h, and 3 and 7 days after ICH; Fig. S1). The treatment groups were as follows: Sham-operated, ICH model, ICH + 3-methyladenine (3-MA), $\mathrm{ICH}+\mathrm{EA}$ (EA group), $\mathrm{ICH}+3-\mathrm{MA}+\mathrm{EA}$ (3-MA + EA group). The experimental process is as illustrated in Fig. S1.

Administration of 3-MA. 3-MA is a PI3K inhibitor that inhibits autophagy at the initiation stage and is used as an inhibitor of mitophagy $(32,37)$. In the present study, 3-MA (400 nm/ $\mu$; Selleck Chemicals) was dissolved in $0.9 \%$ saline by heating to $60-70^{\circ} \mathrm{C}$ until completely dissolved and cooling 
A

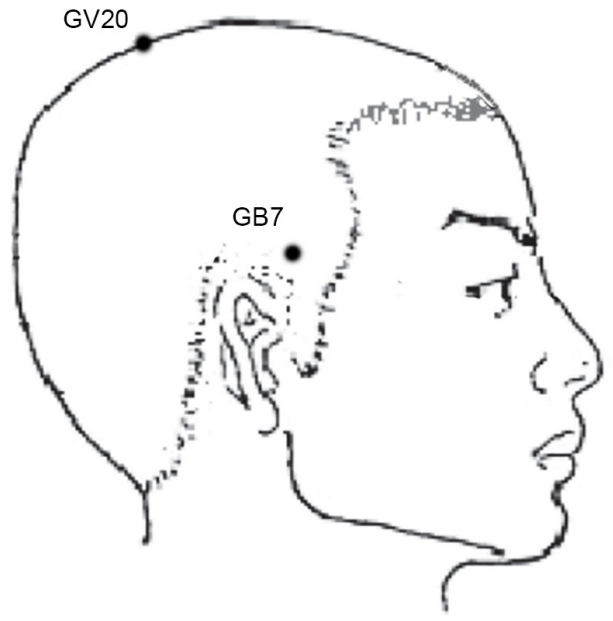

B

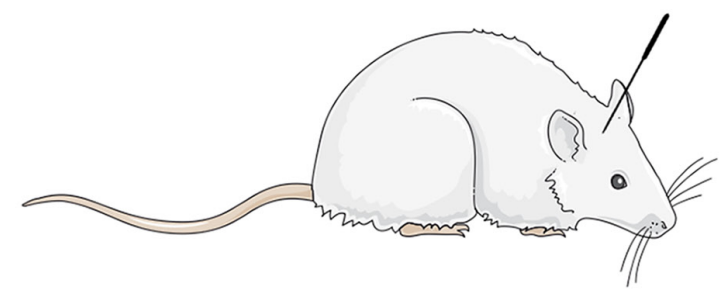

Figure 1. Acupuncture points in mice were determined using a trans-positional method. (A) Location of GV20 and GB7 on humans. (B) Schematic diagram of GV20-GB7 on a rat. Baihui (GV20) is located at the midpoint of the line connecting the apexes of the bilateral ear on the parietal bone; Qubin (GB7) is at the posterior 2/3 point of the line connecting the right orbital margin and porus acusticus externus.

to room temperature immediately before treatment. Rats in the 3-MA and 3-MA + EA groups were anesthetized as aforementioned and placed on stereotaxic apparatus, then $10 \mu 13-\mathrm{MA}$ was injected into the lateral ventricles $(1.5 \mathrm{~mm}$ anterior and $0.8 \mathrm{~mm}$ laterally to the right of bregma) $15 \mathrm{~min}$ before whole blood-induced ICH surgery.

EA treatment. Rats were placed into a rat fixator (Beijing Ji Nuotai Science and Technology Development Co., Ltd.). Treatment was performed $2 \mathrm{~h}$ after the ICH model was established, and once every $24 \mathrm{~h}$ thereafter. Baihui (GV20) was located according to the world acupuncture and moxibustion congress $(38,39)$, and acupoint Qubin (GB7) was determined using a trans-positional method (40) (Fig. 1). Briefly, GB7 was located on the connecting line of the orbital margin and porus acusticus externus. A resistance detector was used to measure the electrical characterization along the proposed line (resistance of acupoints is lower compared with non-acupoints) (41). The posterior $2 / 3$ point of the line connecting the orbital margin and porus acusticus externus was confirmed as the GB7. Sterilized acupuncture needles $(0.25 \times 15.00 \mathrm{~mm}$ Hwato disposable acupuncture needle; Suzhou Medical Products Factory Co., Ltd.) were inserted at Baihui (GV20) with the needle tip penetrating $\sim 8 \mathrm{~mm}$ ipsilateral (focal side; contralateral side of the paralyzed limb) acupoint Qubin (GB7) (Fig. 1). The needle was connected to an EA instrument (model G6805) and EA was performed with continuous-wave at $4 \mathrm{~Hz}$ stimulation for $15 \mathrm{~min} /$ day at an intensity of $1 \mathrm{~mA}$. Rats of all groups were fixed to the fixator in the same way.

Assessment of neurobehavioral deficits. Modified neurological severity score (mNSS) was used to evaluate neurological deficits. mNSS tests were performed as described by Chen et al (42) immediately following recovery from anesthesia, and at 6 and $24 \mathrm{~h}$ and 3 and 7 days following EA treatment. Briefly, the evaluation criteria comprises 18 points as follows: Motor tests, including raising rats by the tail and floor walk tests, 6 points; sensory tests, including placing and proprioceptive tests, 2 points; beam balance test, 6 points (normal=0; maximum $=6$ ); absent reflex and abnormal movement tests, including pinna, corneal and startle reflexes, and seizures, 4 points. The test data were assessed by an experimenter who was blinded to the experimental design and treatment procedure. The total mNSS was classified as follows: 13-18, serious injury; 7-12, moderate injury; 1-6, slight injury. Animals scoring 7-13 points following the operation were considered to indicate a successful ICH model and were selected for neurobehavioral deficit assessment after treatment. These animals exhibited symptoms such as tipping over to the paralyzed side, hind leg buckling, falling from the balance beam and disappearance of corneal reflex.

TEM. Rats were euthanized with pentobarbital $(120 \mathrm{mg} / \mathrm{kg}$, i.p.) after 6 and 24 h, 3 days and 7 days respectively, then perfused with pre-cooled $\left(4^{\circ} \mathrm{C}\right)$ physiological saline, followed by PBS containing $4 \%$ paraformaldehyde. The brain was removed and cut into $1 \mathrm{~cm}^{3}$ cubes, which were fixed with $2.5 \%$ glutaraldehyde for $4 \mathrm{~h}$, washed four times in $0.1 \mathrm{M}$ PBS (pH 7.4) overnight at $40^{\circ} \mathrm{C}$, then postfixed in $1 \%$ osmium tetroxide for $2 \mathrm{~h}$ at $30^{\circ} \mathrm{C}$ and washed with PBS again. Following dehydration by graded alcohol and dry acetone, samples were embedded in Epon/Araldite mixture (Merck KGaA); polymerization was performed at $70^{\circ} \mathrm{C}$ for 2 days. Ultrathin sections $(70 \mathrm{~nm})$ were cut using a Leica Ultracut microtome (Leica Microsystems, Inc.), and stained with $3 \%$ lead citrate and uranyl acetate staining solution for 10 and $30 \mathrm{~min}$ respectively, then drying at room temperature. The samples were observed under a Philips 201 electron microscope (Philips Medical Systems B.V.) and images were captured.

Western blot analysis. Following euthanasia with pentobarbital $(120 \mathrm{mg} / \mathrm{kg}$, i.p.), rats were decapitated and the brain was removed. The focal area was dissected and placed on ice. Subsequently, samples were homogenized in lysis 
buffer (cat. no. M334-100ML; Amresco, LLC), followed by centrifugation at $15,100 \times \mathrm{g}$ for $10 \mathrm{~min}$ at $4^{\circ} \mathrm{C}$. The protein concentration was determined using a BCA protein assay kit (cat. no. WLA004; Wanleibio, Co., Ltd.). Equal amounts of denatured protein $(50 \mu \mathrm{g})$ were separated by SDS-PAGE on $10 \%$ gels, then transferred onto PVDF membranes (EMD Millipore). The membranes were blocked for $2 \mathrm{~h}$ with $5 \%$ non-fat milk in $0.1 \%$ TBS-Tween-20 (cat. no. WLA025. Wanleibio Co., Ltd.) at room temperature and then incubated overnight at $4{ }^{\circ} \mathrm{C}$ with the following primary antibodies: BNIP3 (1:400; cat. no. bs-4239R; BIOSS), PINK1 (1:1,000; cat. no. 23274-1-AP; ProteinTech Group, Inc.), Parkin (1:400; cat. no. WL02512; Wanleibio Co., Ltd.), translocase of outer mitochondrial membrane 20 homolog (1:500; TOMM20; cat. no. WL03626; Wanleibio Co., Ltd.), cytochrome $c$ oxidase IV (1:400; COX IV; cat. no. WL02203; Wanleibio Co., Ltd.), Bcl-2 (1:500; cat. no. 12789-1-AP; ProteinTech Group, Inc.), BAX (1:400; cat. no. WL01637; Wanleibio Co., Ltd.) cleaved caspase-3 (1:300; cat. no. 19677-1-AP; ProteinTech Group, Inc.) and p53 (1:300; cat. no. 10442-1-AP; ProteinTech Group, Inc.). The membranes were washed in TBST and incubated with horseradish peroxidase-conjugated (HRP) secondary goat anti-rabbit IgG (1:5,000; cat. no. 7074; Cell Signaling Technology, Inc.) for $1 \mathrm{~h}$ at $37^{\circ} \mathrm{C}$, then the PVDF membranes were added to TBST bufferat room temperature, and rotated for $15 \mathrm{~min}$. Immunoblotting was detected by electrochemiluminescence (cat. no. WLA003, Wanleibio Co., Ltd.), and densitometry was analyzed using a Bio-image Analysis system (Bio-Rad Laboratories, Inc.). $\beta$-actin (1:1,000; cat. no. WL01845; Wanleibio Co., Ltd.) was used as a loading control. Results were calculated as the ratio of the fluorescence intensity of target protein $/ \beta$-actin.

Immunohistochemistry. Rats were euthanized with pentobarbital $(120 \mathrm{mg} / \mathrm{kg}$, i.p.), perfused as aforementioned and the brain was removed. Paraffin-embedded brain tissue was cut into 4- $\mu \mathrm{m}$ sections. A two-step method of immunohistochemistry was performed according to the instructions of kit (cat. no. PV-6002; ZSGB-BIO, Inc). Briefly, sections were deparaffinized and rehydrated, then incubated in $3 \% \mathrm{H}_{2} \mathrm{O}_{2}$ for $10 \mathrm{~min}$, followed by immersion in $10 \mathrm{mmol} / \mathrm{l}$ citrate buffer ( $\mathrm{pH}$ 6.0) and heating to boil for $2 \mathrm{~min}$ then cooling to room temperature. The sections were then incubated with primary antibodies against p53 (1:300; cat. no. 10442-1-AP; ProteinTech Group, Inc.) overnight at $4^{\circ} \mathrm{C}$. After washing in PBS, sections were incubated with anti-rabbit biotinylated secondary antibody (1:500; cat. no. bs-0295G; BIOSS) at $37^{\circ} \mathrm{C}$ for $30 \mathrm{~min}$, then stained with $4 \%$ diaminobenzidine and $5 \%$ hematoxylin for $30 \mathrm{~min}$ at room temperature. Sections were visualized using a Motic3000 light microscope (Motic Incorporation, Ltd.) at x400 magnification. Cells were counted in five randomly selected fields of view in each group. Nuclei were stained blue and p53-positive cells were visualized as yellow-brown granules.

Immunofluorescence staining. After rats were euthanized with pentobarbital (120 $\mathrm{mg} / \mathrm{kg}$, i.p.) and perfused as aforementioned, brains were collected and immersed in gradient alcohol, followed by immersion in dimethylbenzene. Paraffin-embedded brain tissue was then cut into
5- $\mu \mathrm{m}$ sections. Following deparaffinization, sections were permeabilized with gradient alcohol. The permeabilization solution was then removed and samples were washed three times in PBS for 5 min each. Subsequently, the sections were blocked with $10 \%$ goat serum for $15 \mathrm{~min}$ at room temperature (cat. no. SL083; Beijing Solarbio Science \& Technology Co., Ltd.) and slides were incubated overnight with primary antibody anti-LC3II (1:100; cat. no. bs-2912R; BIOSS) at $4^{\circ} \mathrm{C}$. The sections were washed with PBS and incubated with fluorescein isothiocyanate-labeled goat anti-rabbit antibody (1:200; cat. no. A0516; Beyotime Institute of Biotechnology) for $1 \mathrm{~h}$ at room temperature in the dark. In order to detect the morphology of the nucleus, the nuclei were stained with DAPI $(0.1 \mu \mathrm{g} / \mathrm{ml})$ for $15 \mathrm{~min}$ at room temperature and detected by blue fluorescence. After rinsing with PBS three times (5 min each), the sections were mounted on slides. Confocal images were captured at $\mathrm{x} 400$ magnification using a fluorescence microscope (DP73; Olympus Corporation).

TUNEL. Rats were sacrificed with pentobarbital $(120 \mathrm{mg} / \mathrm{kg}$,i.p.) and perfused as aforementioned, after which, the brains were cut into sections $(10 \mu \mathrm{m})$ and TUNEL assay was performed using a TUNELAP kit (OriGene Technologies, Inc.) according to the manufacturer's instructions. Briefly, the sections were washed with PBS and reacted with TdT enzyme/buffer at $37^{\circ} \mathrm{C}$ for $1 \mathrm{~h}$, followed by enzyme-labeled anti-dUTP antibody reaction at room temperature for $30 \mathrm{~min}$. Then, the sections were rinsed with 3,3-diaminobenzidine and dehydrated with dimethylbenzene. After being air-dried, the sections were observed and images were captured at x400 magnification using a Motic3000 light microscope; six fields of view were quantitatively assessed from each section $(n=6)$ using Image-pro Plus 6.0 Pathological Image Analysis system (Media Cybernetic, Inc.). Brown staining of nuclei was considered to indicate apoptotic cells.

Statistical analysis. Data are presented as the mean \pm SEM $(n=5)$. P-values for western blotting, immunohistochemistry and TUNEL assay were determined by one-way ANOVA followed by Tukey's post hoc test. Data are presented as median (IQR). P-values for mNSS were determined by Kruskal-Wallis test, followed by post hoc Dunn's multiple comparisons test. GraphPad Prism 8.0 software (GraphPad Software, Inc.) was used for all histograms and statistical analysis. $\mathrm{P}<0.05$ was considered to indicate a statistically significant difference.

\section{Results}

Mitophagy is activated following ICH. The induction of mitophagy following ICH was evaluated by detecting the protein expression levels of BNIP3, a protein that carries only one Bcl-2-homology-3 domain of the Bcl-2 family and mitochondrial autophagy receptor (43). Immunoblotting showed ICH caused a time-dependent increase in BNIP3 (Fig. 2A). Semi-quantitative analysis of protein band density indicated that BNIP3 protein expression levels increased at $6 \mathrm{~h}$ after ICH compared with the sham group and peaked at 3 days. The expression levels on day 7 after ICH induction were still higher compared with the control group (Fig. 2B). Mitophagy protein 
A

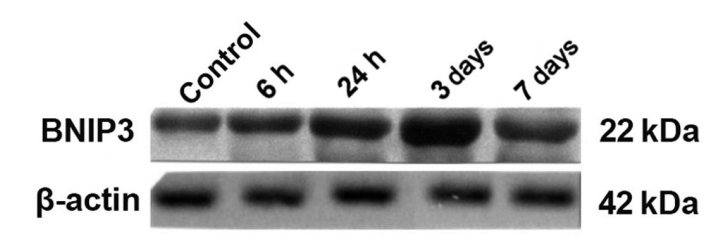

C

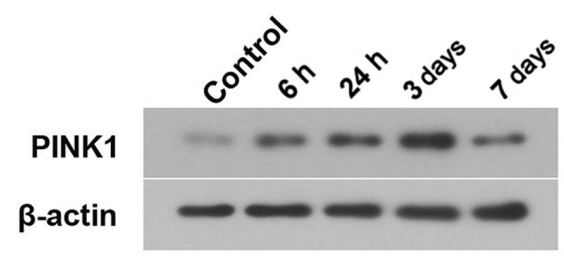

E

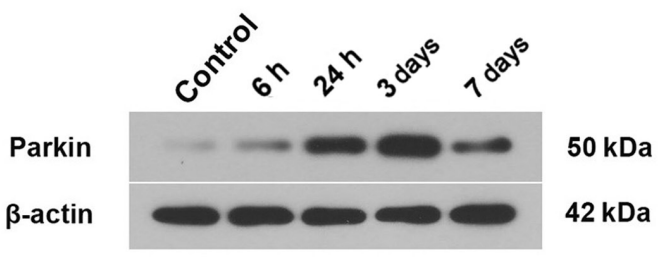

B

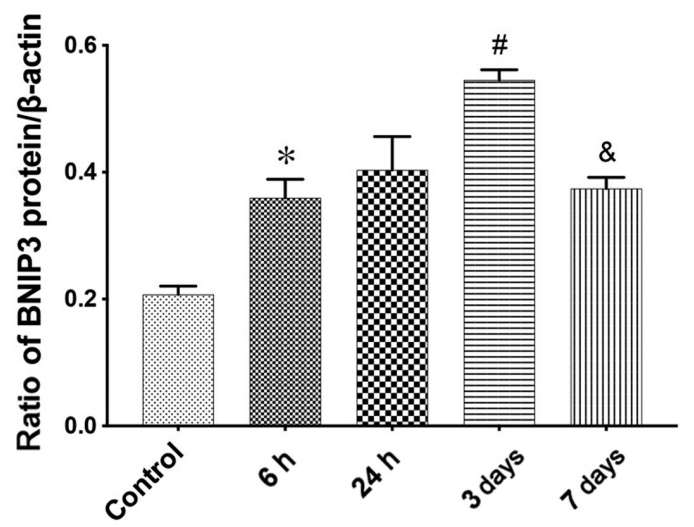

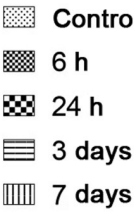

\section{$45 \mathrm{kDa}$ \\ $42 \mathrm{kDa}$}

F
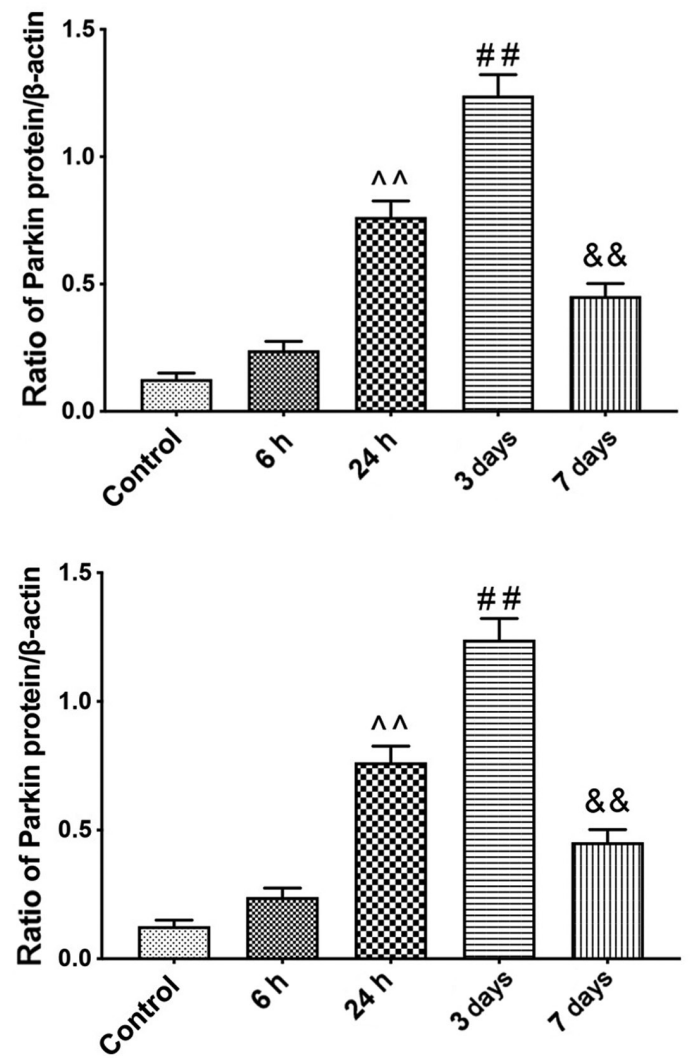

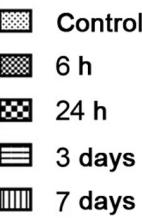

血 7 days

Figure 2. Mitophagy is activated following intracerebral hemorrhage. Expression levels and semi-quantitative analysis of (A and B) BNIP3, (C and D) PINK1 and (E and F) Parkin, as determined by western blot analysis. $\beta$-actin protein was used as the loading control. Data are expressed as the mean \pm SEM ( $\mathrm{n}=5$ /group) and analyzed by one-way ANOVA followed by Tukey's multiple comparisons test. ${ }^{*} \mathrm{P}<0.05$ vs. Control; ${ }^{\wedge} \mathrm{P}<0.01$ vs. 6 h; $\mathrm{P}<0.05$, ${ }^{\# \prime} \mathrm{P}<0.01$ vs. $24 \mathrm{~h}$; ${ }^{\&} \mathrm{P}<0.05,{ }^{\& \&} \mathrm{P}<0.01$ vs. 3 days. BNIP3, BCL/adenovirus E1B 19k Da-interacting protein 3; PINK1, PTEN-induced putative kinase 1.

markers PINK1 and Parkin were also detected by western blotting to determine mitophagy activity following ICH; the results were consistent with those of BNIP3 (Fig. 2C-F).

Autophagy following ICH was identified by immunofluorescent techniques. LC3-II-positive cells were notably increased at all time points in the ICH group compared with the control group, indicating autophagy was activated (Fig. 3). The results showed that the lighteness of LC3-II positive neurons (Fig. 3) at day 3 following ICH was significantly higher than that in other groups. The observed LC3-II-positive neurons were notably decreased at 7 days following ICH. (Fig. 3A).

The ultrastructural phenotypes of autophagosomes, autolysosomes and mitochondria were investigated by TEM (Fig. 3B). A large number of healthy mitochondria with clear crista structures were observed in the Control group (Fig. 3B-a). Double-membrane autophagosomes containing mitochondria, which are a typical morphological characteristic of mitophagy, were observed following ICH (Fig. 3Bb-e). Greater numbers of these autophagosomes and autolysosomes were observed at day 3 following ICH, indicating increased mitophagy (Fig. 3B-d). Damaged and deformed mitochondria with vague cristae and lost matrix granules were also observed following ICH. These results indicated that mitophagy was activated following ICH; this effect was most notable at 3 days after ICH (Fig. 3B-b-e).

EA treatment upregulates mitophagy following ICH. The effect of EA on mitophagy following ICH was assessed by 
A

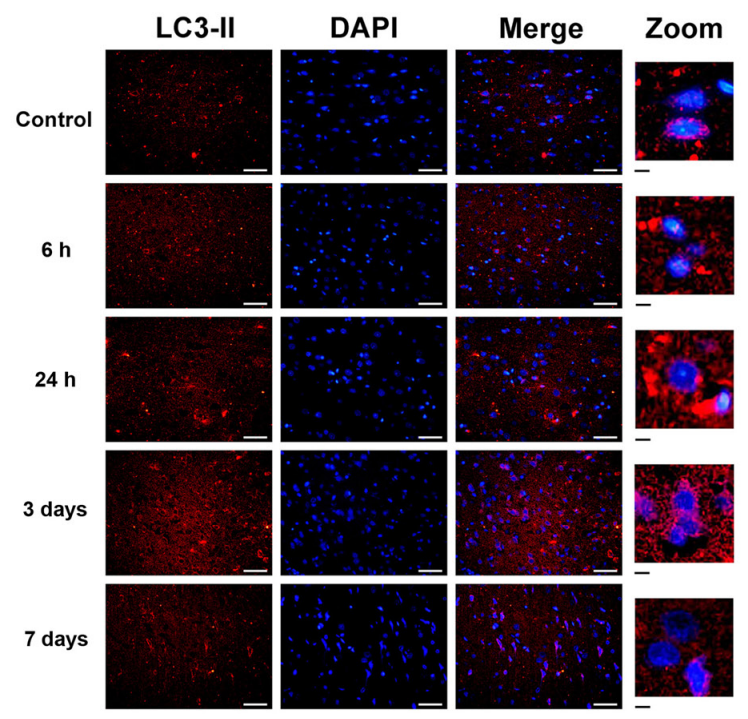

B

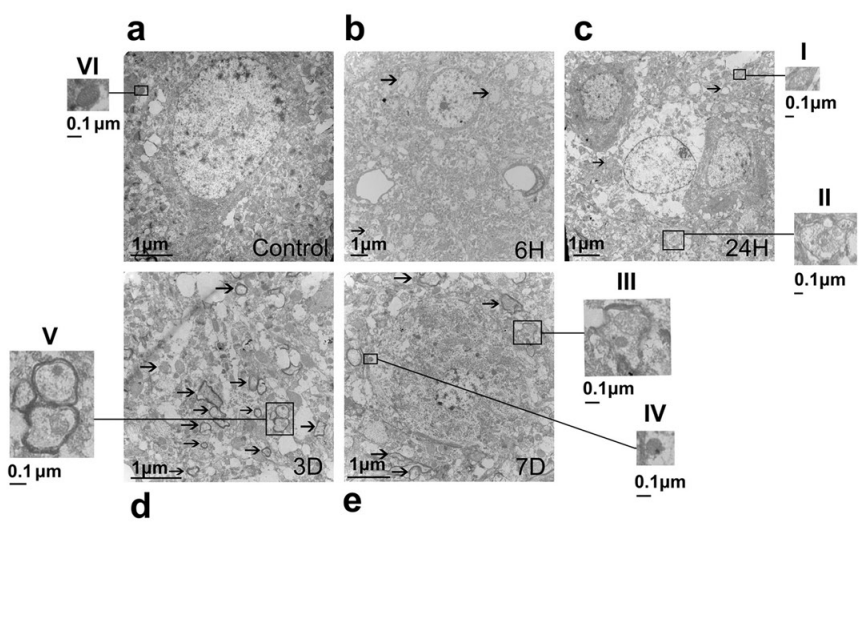

Figure 3. Autophagy is activated following ICH. (A) Representative immunofluorescence images of LC3-II (red) surrounding the basal ganglia at different time points following ICH. More LC3-II puncta were observed on day 3 compared with other time points. Scale bar, $50 \mu \mathrm{m}$. Magnified scale bar, $5 \mu \mathrm{m}$. (B) Transmission electron microscopy analysis of ultrastructural changes at different time points following ICH. (a) Healthy mitochondria with clear crista structure were observed in the sham operation group. Magnification, x10,000. (b-e) Morphological changes in the basal ganglia of model groups at different time points. Black arrows indicate double-membrane autophagic vacuoles, including partially degraded mitochondria or other autophagic content. Magnification, x4,000, x6,000, x12,000 and x12,000, respectively. I, Large mitochondrion with disordered cristae; II, autolysosome containing residues of mitochondria and lytic organelles; III and V, autophagosomes containing mitochondria, demonstrating ongoing mitophagy; IV, lysosome; VI, healthy mitochondrion with normal size and clear crista structure.

measuring BNIP3 expression levels (Fig. 4A and B). The expression levels of BNIP3 were increased following ICH and significantly increased in the EA group at 7 days compared with the model group. Compared with the model group, the 3-MA group exhibited lower expression levels of BNIP3 in the focal area at 7 days. Notably, the effects of EA (GV20-GB7) were blocked by 3-MA.

The relative number of mitochondria at day 3 following ICH was assessed by levels of mitochondrial protein markers TOMM20 and COX IV (Fig. 4C and D). Expression levels of both TOMM20 and COX IV increased following ICH; this was alleviated by EA treatment. Treatment with 3-MA increased the expression levels of TOMM20 and COX IV compared with the Model group (Fig. 4E and F), indicating inhibition of mitophagy. These results suggested that EA treatment may enhance autophagy-associated mitochondrial elimination following ICH.

TEM was performed to investigate the effect of EA on stimulating focal mitophagy. Since mitophagy was most activated on day 3 after ICH, this time point was selected for the TEM experiments. There was no notable observation of autophagic vacuoles in the sham group but autophagosomes and autolysosomes containing mitochondria were observed in the model group. Swollen and dilated mitochondria and rough endoplasmic reticulum were also observed, compared with the sham group (Fig. 5A and B). Larger numbers of autophagosomes with mitochondria and autolysosomes with partially degraded mitochondria, which are characteristic of mitophagy, were observed in the electron micrographs from the EA group, but mild neuron injury was still visible (Fig. 5C). Morphological changes were found in the 3-MA group, including decreased numbers of autophagosomes, shrunken nuclei and disrupted cell membranes, which indicated early-stage apoptosis (Fig. 5D). In addition, treatment with 3-MA neutralized the effect of EA on mitophagy enhancement (Fig. 5E). These results indicated that inhibiting mitophagy may lead to neuron apoptosis; enhancing mitophagy via EA decreased accumulation of injured mitochondria, which may delay neuron death. Collectively, these results demonstrated that mitophagy was augmented by EA (GV20-GB7).

EA decreases mitochondrial apoptosis following ICH. The anti-apoptotic protein Bcl-2 is activated in mitochondrial apoptosis (44). In the present study, Bcl-2 was upregulated at $6 \mathrm{~h}$ following $\mathrm{ICH}$ in the model group compared with the sham group (Fig. 6A and B) and declined after $24 \mathrm{~h}$ to reach a minimum level 3 days after ICH. EA treatment significantly increased $\mathrm{Bcl}-2$ expression relative to the model group.

Cleaved caspase-3, an executioner protease of apoptosis, was also detected in the present study (Fig. 6C and D). Cleaved caspase-3 expression was increased following $\mathrm{ICH}$, whereas EA (GV20-GB7) treatment decreased cleaved caspase-3 expression levels at 6 and $24 \mathrm{~h}$.

Bax was also detected by western blot analysis (Fig. 6E and F). The expression of Bax was upregulated after ICH was initiated and enhanced by EA treatment compared to the Model group. Additional 3-MA treatment reversed the effects of EA; in addition, 3-MA decreased Bcl-2 expression levels, and increased those of Bax and cleaved caspase- 3 compared with the model group. Notably, cleaved caspase- 3 peaked at $24 \mathrm{~h}$ after $\mathrm{ICH}$, which may be due to the apoptotic function of cleaved caspase- 3 in the early stages after stroke $(45,46)$. TUNEL assay demonstrated that the number of apoptotic cells increased in ICH rats. Apoptosis was reduced 
A

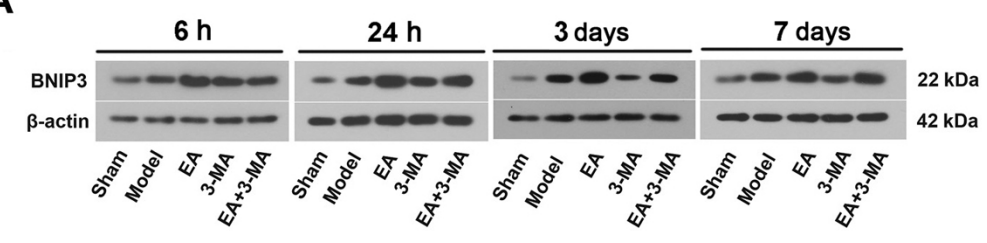

C

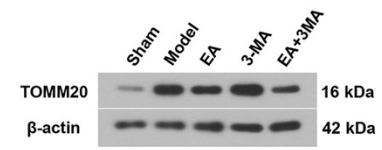

E

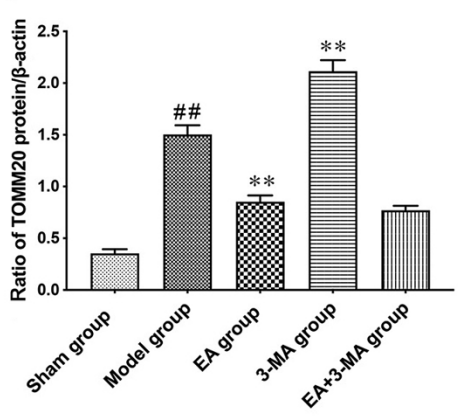

Sham group Model group

囷 EA group

目 3-MA group

皿 EA+3-MA group
B

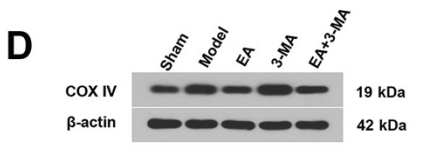

F

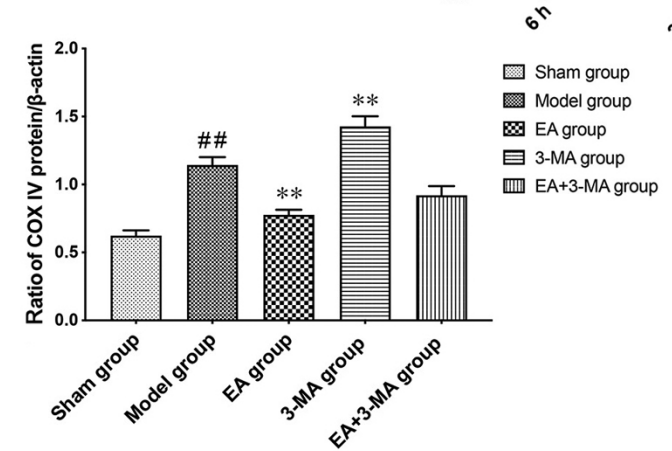

Sham group

in Model group

\& EA group

目 3-MAgroup

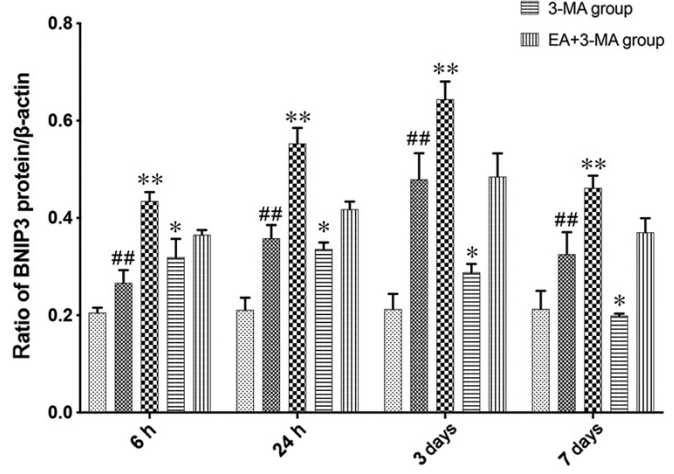

Figure 4. EA treatment augments mitophagy following ICH. Mitophagy in each group following ICH was evaluated by western blotting and semi-quantitative analysis of (A and B) BNIP3, (C and E) TOMM20 and (D and F) COX IV. $\beta$-actin was used as a loading control. Data are presented as the mean \pm SEM (n=5/group). ${ }^{\# \#} \mathrm{P}<0.01$ vs. Sham; ${ }^{*} \mathrm{P}<0.05,{ }^{* *} \mathrm{P}<0.01$ vs. Model. ICH, intracerebral hemorrhage; BNIP3, BCL/adenovirus E1B 19 kDa-interacting protein 3; TOMM20, translocase of outer mitochondrial membrane 20 homolog; COX IV, cytochrome $c$ oxidase IV; EA, electroacupuncture; 3-MA, 3-methyladenine.

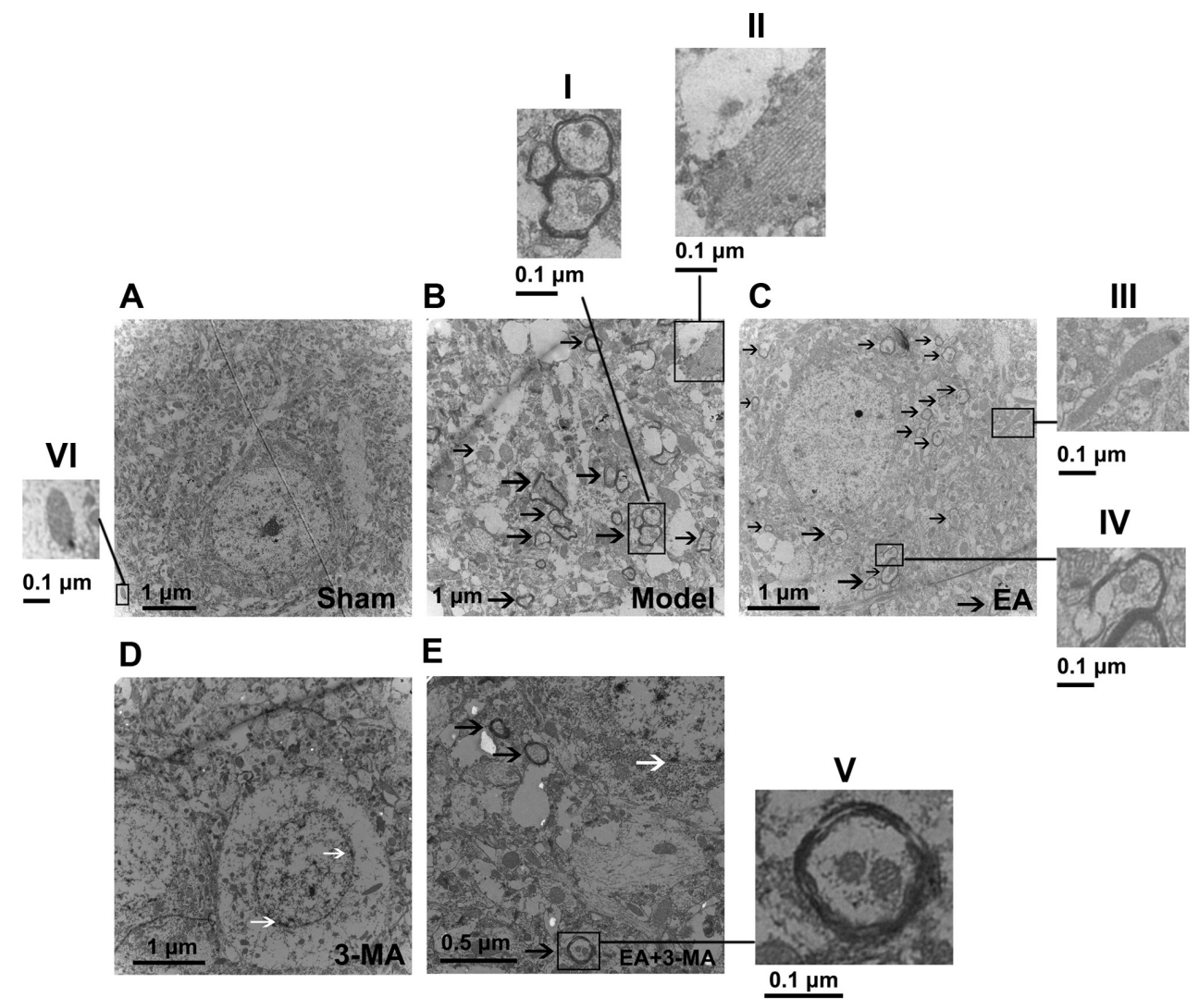

Figure 5. Transmission electron microscopy images of focal bleeding areas of rats in each group. Images were captured at (A) x5,000, (B) x12,000, (C and D) x7,000 and (E) x15,000 magnification. Black arrows indicate double-membrane autophagic vacuoles, which include partially degraded mitochondria or other autophagic content. White arrows indicate disrupted cell membranes. I and V, Typical mitophagic mitochondrion encapsulated by double-membrane autophagosomes; II, rough endoplasmic reticulum; III, deformed mitochondrion; IV, autolysosome; VI, normal mitochondrion with tight, orderly cristae. 
A

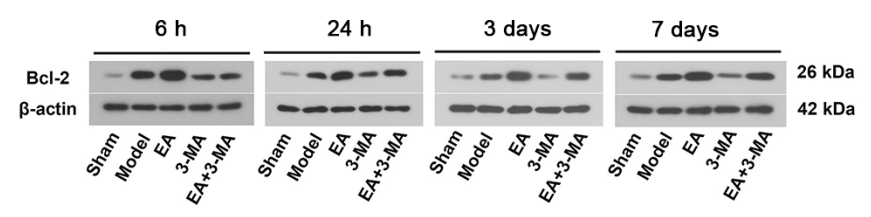

C

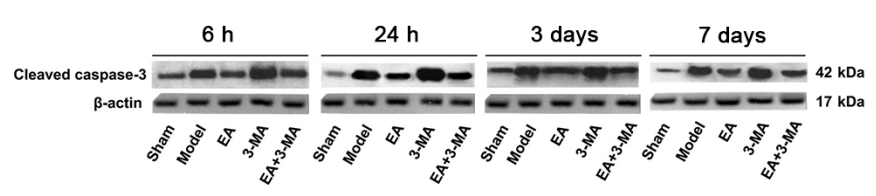

E

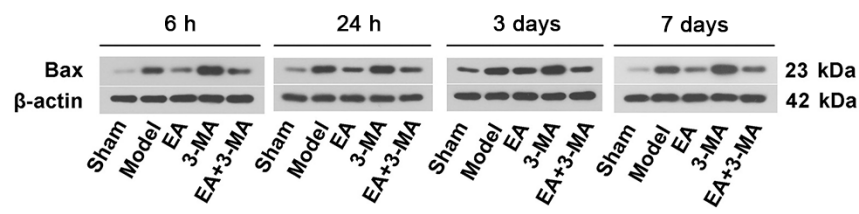

G

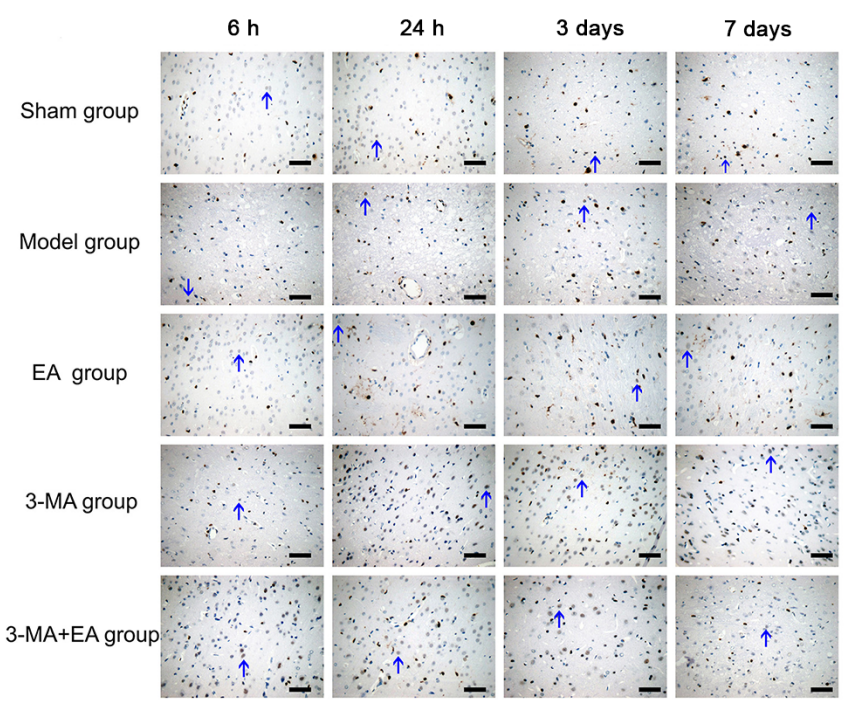

B

D

$\mathbf{F}$
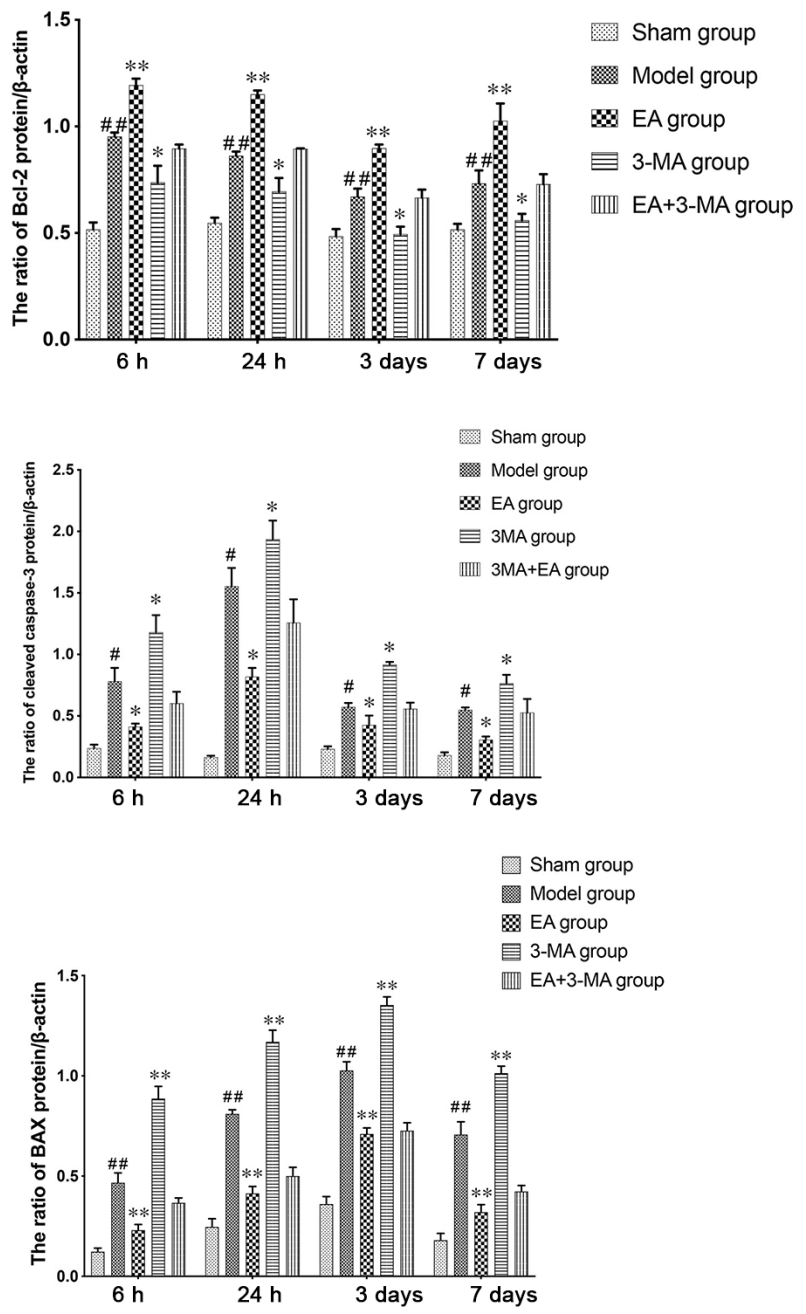

H

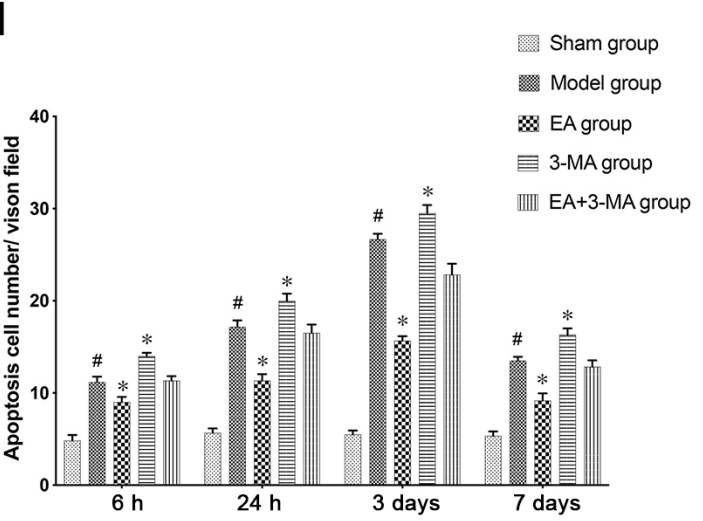

Figure 6. EA treatment decreases apoptosis following intracerebral hemorrhage. Expression levels and relative band density of (A and B) anti-apoptotic protein Bcl-2, (C and D) cleaved caspase- 3 and (E and F) Bax in each group were determined by immunoblotting. $\beta$-actin was used as the internal control. Data are presented as the mean \pm SEM ( $\mathrm{n}=5$ /group). (G) Apoptotic cells were evaluated by TUNEL staining. Blue arrows indicate brown-stained TUNEL-positive cells Apoptotic cells were counted in six randomly selected fields of view at a magnification of $\mathrm{x} 400$. Scale bar, $50 \mu \mathrm{m}$. (H) Semi-quantitative analysis of TUNEL staining of apoptotic cells. ${ }^{\#} \mathrm{P}<0.05,{ }^{\# \#} \mathrm{P}<0.01$ vs. Sham; ${ }^{*} \mathrm{P}<0.05,{ }^{* *} \mathrm{P}<0.01$ vs. Model. EA, electroacupuncture; 3-MA, 3-methyladenine.

by EA treatment, whereas increased by administration of 3-MA, of which in accordance with the western blotting results (Fig. 6G and $\mathrm{H}$ ).

EA suppresses expression of $p 53$. Tumor suppressor protein p53 regulates apoptosis by interacting with both anti- and pro-apoptotic Bcl-2 family proteins (47). Activation of the p53 system has also been found to induce autophagy (48). In the present study, the expression levels of p53 were evaluated by western blot analysis (Fig. 7A and B); the expression levels of p53 in the focal area were significantly elevated at $6 \mathrm{~h}$ after ICH, peaked at 3 days and remained high at 7 days. EA treatment significantly prevented the elevation of p53 levels compared with the model group at each time point, whereas 
A

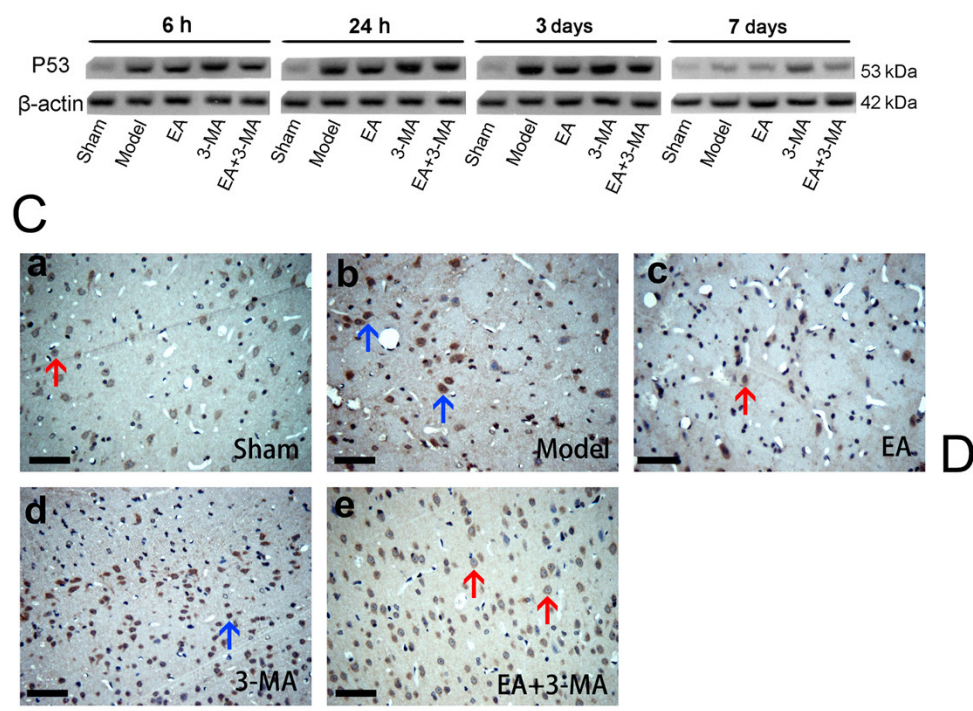

B
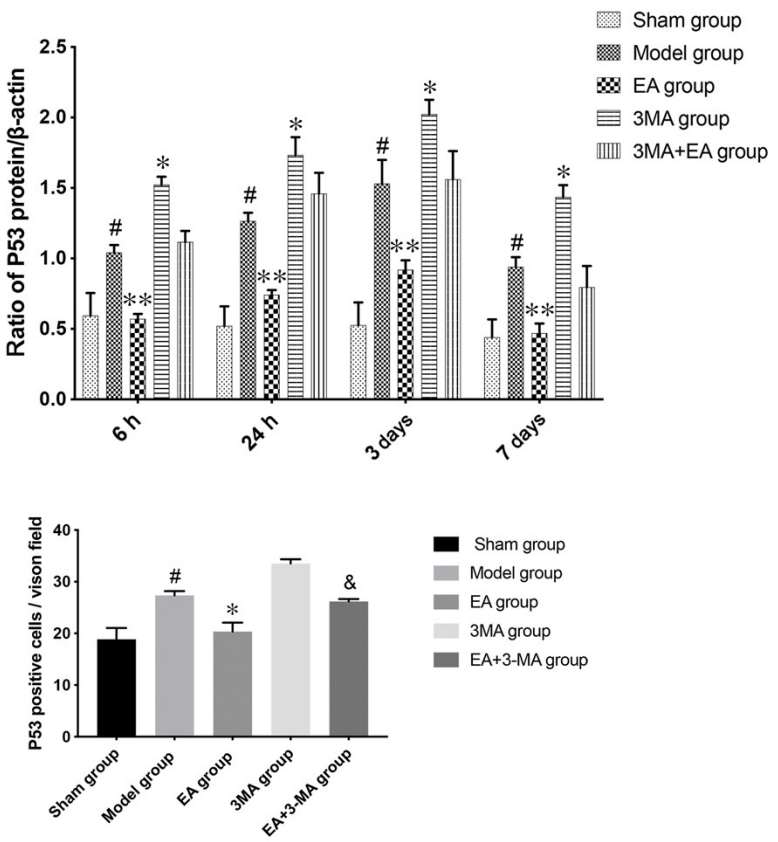

Figure 7.EA treatment decreases expression levels of p53 in neuronal cytoplasm.(A) Western blotting demonstrated expression levels of p53. (B) Semi-quantitative analysis of p53 expression levels. $\beta$-actin was used as the internal control. Data are presented as the mean \pm SEM (n=5/group) and analyzed by one-way ANOVA followed by Tukey's multiple comparison test. (C) p53 immunohistochemical staining of basal ganglia hemorrhage area at 3 days. (a) The expression of p53 in the Sham group; (b) more expression of p53 was observed in Model group; (c) EA treatment decreased mainly cytoplasmic expression levels of p53 in neurons; (d) 3-MA treatment increased the p53-positive signals; (e) treatment of 3-MA neutralized the effect of EA. Tissue samples were observed under light microscopy at a magnification of $\mathrm{x} 400$. Red and blue arrows indicate positive signals of p53 in the cytoplasm and nucleus, respectively. Scale bar, $50 \mu \mathrm{m}$. (D) Semi-quantitative analysis of p53-positive cells. ${ }^{\#} \mathrm{P}<0.05$ vs. Sham; ${ }^{*} \mathrm{P}<0.05,{ }^{* *} \mathrm{P}<0.01$ vs. Model; ${ }^{*} \mathrm{P}<0.05$ vs. EA. EA, electroacupuncture; 3 -MA, 3-methyladenine.

3-MA promoted p53 expression and counteracted the effect of EA treatment. Immunohistochemical analysis was performed to confirm the distribution of p53 (Fig. 7C and D); numbers of p53-positive cells were consistent with the results of western blot analysis. Positive signals of p53 were strongly deposited in the cytoplasm of neuronal cells on day 3 after ICH.

Neurobehavioral deficits following ICH. Neurobehavioral deficits were evaluated to investigate the effect of EA on recovery and mitophagy following $\mathrm{ICH}$. Neurobehavioral deficits were assessed by mNS tests at 6 and $24 \mathrm{~h}$ and 3 and 7 days after $\mathrm{ICH}$ in the presence or absence of EA treatment. Rats in each group exhibited neurological deficits at $6 \mathrm{~h}$ following induction of $\mathrm{ICH}$, which improved over time, as indicated by decreased mNSS test scores (Fig. 8). However, mNSS in the EA group showed no significant difference compared with the model group until day 7 after EA treatment (Fig. 8). Alleviation of neurological deficits was counteracted by 3 -MA.

\section{Discussion}

Mitochondrial dysfunction is associated with the pathogenesis of stroke (49). The autophagic degradation of dysfunctional mitochondria is commensurate with mitophagy, which is a mechanism of mitochondrial quality control and is key for maintaining intracellular homeostasis (50). Previously, mitophagy has attracted attention as a novel therapeutic target in stroke $(51,52)$. It has been extensively researched in ischemic stroke (53-55), but there are few studies in the context of $\mathrm{ICH}$.
Scalp acupuncture (SA) is a specialized acupuncture technique used to treat patients with ischemic stroke (56). Baihui (GV20) to Qubin (GB7) is one of the most effective stimulation lines or areas (57). Although SA treatment for acute ICH has been disputed (58), clinical trials have proved its efficacy and safety (59). A randomized controlled study demonstrated that SA, combined with conventional treatment, increased the hematoma absorption ratio and alleviated neurological impairment compared with conventional treatment alone (60). A clinical study by Sun et al (61) of 500 cases of patients with cerebrovascular hemiplegia treated via acupuncture on Baihui-Qubin achieved a satisfactory therapeutic effect. A previous study involving Baihui-Qubin (GV20-GB7) treatment suggest inhibited Notch-Hes signaling pathway transduction in rat basal ganglia after $\mathrm{ICH}$, thereby inhibiting neuronal differentiation and maintaining neural stem cell proliferation (28). The present study focused on investigating the underlying mechanism of the neuroprotective effect of GV20-GB7 EA on mitophagy following ICH.

The PI3K inhibitor 3-MA is an autophagy inhibitor that works in the early stage to suppress autophagy (62). 3-MA has been administered intracerebroventricularly in a number of studies of brain disease $(63,64)$; it has also been administered i.p. in a number of studies on pulmonary and hepatic disease $(65,66)$, but few studies have assessed its impact on cerebral disease $(67,68)$. In addition, the standard dosage of i.p. and intraventricular injection was different in previous studies (69); intraperitoneally injected 3-MA was 150-300 $\mu \mathrm{g} / \mathrm{kg}$, whereas intraventricularly injected 3-MA was 3-10 $\mu \mathrm{l} / \mathrm{rat}$, which was notably lower. Further studies are 
A

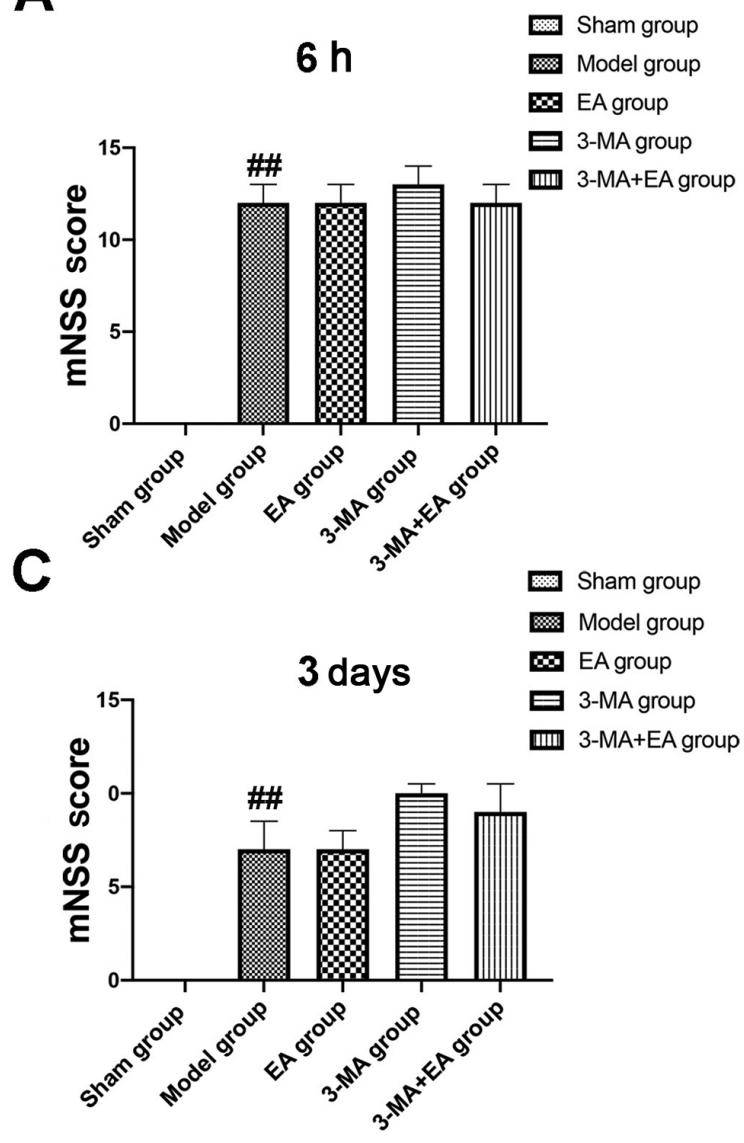

B

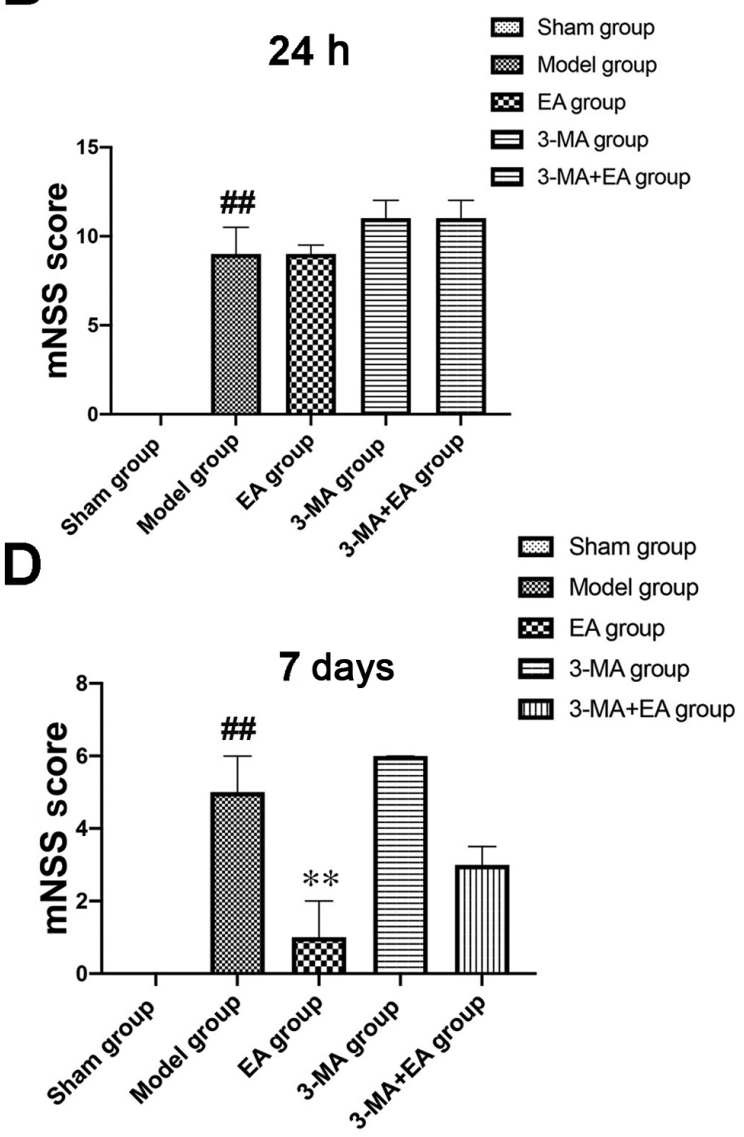

Figure 8. Assessment of mNSS following intracerebral hemorrhage. EA attenuated neurological deficit at 7 days. Data are presented as median (IQR), assessed via Kruskal-Wallis followed by post hoc Dunn's multiple comparison test. EA does not show a significant improvement of the neurological deficit after ICH by at (A) 6 and (B) $24 \mathrm{~h}$ and (C) day 3. (D) EA treatment decreased mNSS at day 7 . ${ }^{* *} \mathrm{P}<0.01$ vs. Model; ${ }^{\# \#} \mathrm{P}<0.01 \mathrm{vs}$. Sham. mNSS, modified neurological severity score; EA, electroacupuncture; 3-MA, 3-methyladenine.

required to determine the effect of this. Evidence has indicated the role of 3-MA as an inhibitor of mitophagy $(70,71)$; to the best of our knowledge, high mortality rate caused by intraventricular 3-MA treatment has not previously been reported. Accordingly, cella lateralis injection of 3-MA was selected in the present study.

In the present study, evidence of autophagy was detected in focal tissue by observing the expression of LC3-II; mitophagy was reflected by the expression levels of mitophagy protein markers, such as PINK1, Parkin and BNIP3, which indicated that mitophagy increased gradually following $\mathrm{ICH}$ and peaked at day 3. In order to investigate whether mitophagy was triggered following $\mathrm{ICH}$, morphological changes of neurons were observed by TEM. Autophagic vacuoles appeared shortly following ICH; lysosomes and degraded mitochondria were also detected, indicating that mitochondrial autophagy was activated.

The efficient autophagic degradation of mitochondria has been reported to require the participation of BNIP3, thus indicating that mitophagy is specifically activated by BNIP3 (72). BNIP3 is a pro-apoptotic member of the Bcl-2 family with an atypical $\mathrm{BH} 3$ domain, which can also function as a receptor for targeting autophagosomes to mitochondria $(73,74)$. Due to its links with both mitophagy and apoptosis (75), BNIP3 was selected in the present study as a target to investigate changes in mitophagy following ICH. EA treatment promoted mitophagy by upregulating BNIP3; the therapeutic effect was mitigated by 3 -MA. Inhibition of expression of BNIP3 or mitophagy by 3-MA may be due to the blockage of the LC3-interacting region of BNIP3, which facilitates a direct interaction between BNIP3 and LC3 to promote clearance of dysfunctional mitochondria (72). The present study demonstrated that inhibition of mitophagy by 3-MA may enhance mitochondrial accumulation, as evidenced by decreased levels of the mitophagy marker BNIP3, and increased levels of mitochondrial markers TOMM20 and COX IV in the ICH + 3-MA group after 3 days. The results also suggested that the effect of EA treatment may be achieved by enhancing autophagic clearance of damaged mitochondria, which was consistent with a study by Zhang et al (51). The protective role of autophagy during the reperfusion phase following ischemic stroke may be attributable to mitophagy-associated mitochondrial clearance and inhibition of downstream apoptosis, reflected by decreased COX IV and TOMM20 and increased LC3-II/GAPDH following oxygen-glucose deprivation/reperfusion, 3-MA treatment also increases infarct volume (51). These results indicated that autophagic mitochondrial recycling may serve a protective role following $\mathrm{ICH}$.

Evidence has indicated that mitophagy and apoptosis are interconnected (76,77). Bcl-2 family proteins and p53 
serve dual roles in regulating autophagy and apoptosis (78), yet $\mathrm{p} 53$ and $\mathrm{Bcl}-2$ alteration mechanisms are found to overlap. As a pro-apoptotic protein with homology to Bcl-2 in the $\mathrm{BH} 3$ domain, BNIP3 is considered to be a regulator of mitophagy (79). Previous studies demonstrated that p53 may function primarily as a transrepressor of BNIP3 to protect against hypoxia-induced apoptotic cell death both in vitro and in vivo, suggesting that BNIP3 is associated with dysfunction of mitochondria and neuronal death induced by stroke $(80,81)$. Taken in conjunction with these earlier reports, the present results supported an ICH model in which BNIP3 and p53 modify their activity in a negative feedback loop; EA (GV20-GB7) enhanced mitophagy by upregulating BNIP3, while decreased p53 contributed to a neuroprotective effect.

In order to investigate whether increased mitophagy by EA (GV20-GB7) could serve a neuroprotective role against ICH stress, the effects of increased mitophagy on apoptosis were evaluated. The results showed that, in parallel with the alterations in BNIP3 levels and ultrastructural changes of mitophagy, EA (GV20-GB7) may also inhibit apoptosis by upregulating Bcl-2, as well as decreasing the expression levels of cleaved caspase- 3 and Bax. Previous research has revealed that the caspase and $\mathrm{Bcl}-2$ families jointly participate in the intrinsic apoptosis pathway mediated by mitochondria $(50,82)$. In addition, mitochondrial dysfunction may cause the rupture of the outer mitochondrial membrane and cytochrome $c$ release, which could activate caspase-3 release (83). The potential mechanism may be due to BNIP3 acting as a dual regulator of $\mathrm{Bcl}-2$ family proteins and suppressing apoptosis by inhibiting pro-apoptotic protein $\mathrm{Bcl}-2$ and activating $\mathrm{Bax}$ protein, as well as harboring an LC3-interacting region that can initiate mitophagy $(43,72)$. Combined with the present results, it was suggested that mitophagy enhanced by EA (GV20-GB7) may have an effect on degradation of apoptotic cell death after ICH. However, the mechanism underlying the effects of EA (GV20-GB7) on mitophagy and apoptosis remains unclear. In addition, in test of cleaved caspase- 3 of western blot of this study, the EA and EA + 3-MA group only differed at $24 \mathrm{~h}$. Since 3-MA primarily functions to inhibit mitophagy, the neutralizing effect of 3-MA on EA-induced downregulation of cleaved caspase-3 may differ from that on other factors, such as BNIP3. This may be because the apoptotic function of cleaved caspase- 3 is only active in the early stages following stroke. Expression levels of cleaved caspase-3 were low on day 3 and 7. During the stages of low expression by the day 3 and 7, either EA or 3-MA treatment may not have an obvious effect on the expression levels of cleaved caspase- 3 . Therefore, 3-MA only significantly reversed the effect of EA treatment at $24 \mathrm{~h}$.

The tumor suppressor and transcription factor p53 triggers apoptosis and modulates autophagy by acting at the mitochondrial level $(84,85)$. During the neuronal apoptosis process, p53 has been reported to act as a key factor in early apoptotic signaling in neurons acting upstream of mitochondrial damage and may cause mitochondrial membrane disruption, which can lead to caspase activation (86). As a master regulator of autophagy, the inhibition of autophagy by $\mathrm{p} 53$ has been shown to be associated with its nuclear-to-cytosolic redistribution (87).
Cytoplasmic p53 may translocate to the mitochondria and induce mitochondrial membrane permeabilization by activating pro-apoptotic Bcl-2 family members to inhibit anti-apoptotic Bcl-2 family proteins $(88,89)$. In the present study, EA(GV20-GB7) treatment increased the expression of anti-apoptosis Bcl-2 protein and downregulated the expression of cleaved caspase- 3 and Bax; these results coincided with a significant decrease of p53. From these results, it was concluded that $\mathrm{ICH}$-induced p53 expression in rats was decreased by EA (GV20-GB7), which inhibited apoptosis. In addition, immunohistochemical results showed that p53 was primarily distributed in the cytoplasm, which illustrated that the mitophagy-augmenting and apoptosis-restraining effects of EA treatment may be associated with interactions between mitophagy and apoptotic factors, and downregulated cytoplasmic expression levels of p53 protein. Moreover, 3-MA inhibited mitophagy and abolished the effects of EA, further indicating that in this study, the protective mechanism of EA may involve mitophagy.

In the present study, anti-apoptotic $\mathrm{Bcl}-2$ protein was decreased in response to $\mathrm{ICH}$, meanwhile, the pro-apoptotic protein BNIP3 was increased; however increased BNIP3 also improved mitophagy, which inhibited apoptotic cell death. As p53 is a regulator of both autophagy and apoptosis, it may be suggested that p53 induced apoptosis by interacting with Bcl-2 family proteins, simultaneously inhibiting anti-apoptotic proteins, such as Bcl-2, and activating pro-apoptotic proteins, such as Bax and Bak. BNIP3 may be a putative transcriptional target and downstream effector of p53 in autophagy (90); BNIP3 is also an autophagy (specifically mitophagy) inducer which has similar effects to $\mathrm{p} 53$ when acting as a pro-apoptotic protein. Therefore, EA-alleviated p53 may improve mitophagy and inhibit apoptosis by increasing the expression levels of BNIP3 and decreasing those of Bcl-2.

Neurobehavioral deficits were evaluated by mNSS tests. The behavioral effect of EA was assessed by multiple modalities of testing, including function, sensory (including placing and proprioceptive), beam balance, reflex absence and abnormal movement tests. In the present study, neurobehavioral deficits were not significantly alleviated until day 7 after EA treatment, which is consistent with our previous research $(29,36)$, further suggesting that the benefit of EA increases with treatment duration. Consistent with our previous research (29), each sub-test was observed independently, excluding vibrissae proprioception and symmetry of limb, the assessment failed to demonstrate significantly improved performance by GV20-GB7 EA treatment until 7 days after ICH. Longer observation time and a larger sample size should be used in future studies to improve the validity of the test. EA at GV20-GB7 may improve neurological function following ICH by clearance of mitochondria via mitophagy.

BNIP3 has a C-terminal transmembrane domain, which is essential for its homodimerization and pro-apoptotic function. Following homodimerization, BNIP3 is associated with mitochondrial targeting where it functions in inner membrane depolarization and permeabilization of the mitochondrial outer membrane to induce mitochondrial dysfunction, and both non-apoptotic and apoptotic cell death (91). A study of mouse hepatocytes indicated that hypoxia may increase 
formation of the BNIP3 homodimer but decrease the amount of the monomeric form of BNIP3 (92). Another study also suggested that the BNIP3 homodimer may function as an autophagy receptor to facilitate the removal of organelles, such as mitochondria and endoplasmic reticulum (93). This feature is suggested to serve a wide role in the pathogenesis of various diseases, including stroke. However, to the best of our knowledge, the mechanism underlying activation of dimerized BNIP3, its translocation to the mitochondria during mitophagy, and its potential regulation by acupuncture or other medical intervention have not yet been studied in an ICH model.

There are certain limitations to the present study. All samples used were brain homogenates; thus, the point of mitophagy activation and regulation by p53 cannot be precisely located. Also, the underlying mechanism of EA-enhanced mitophagy was not described here and merits further investigation. Furthermore, it is difficult to conclude whether the effectiveness of EA on mitophagy following ICH was achieved by regulating mitophagy induced by ischemia during ICH secondary injury or by improving mitochondrial metabolism.

Regulation of mitophagy to maintain the integrity and homeostasis of mitochondria is a promising therapeutic target in the future of ICH treatment but further studies are required to reveal the potential mechanism underlying mitophagy following ICH. For example, the exact mechanism by which PINK1 phosphorylates itself and its substrates has not yet been fully elucidated. Parkin recruitment and activation during mitophagy involves PINK1-mediated phosphorylation of Parkin and E3-ubiquitin (94). GTPase mitofusin 2 functions as a mitochondrial receptor for Parkin, which can mediate Parkin recruitment to mitochondria and can further promote Parkin-mediated ubiquitination by p-PINK1 (95). Considering the importance of healthy mitochondria for recovery and survival following ICH (96), mitophagy is an important research area for the development of novel treatments and to reveal how EA may improve neurobehavioral deficits.

In conclusion, the present study identified the role of mitophagy in an ICH model. The neuroprotective effect of EA on Baihui-Qubin (GV20-GB7), which occurs via enhanced mitophagy, may be induced by inhibiting apoptosis. The protective effect of p53 inhibition by EA on GV20-GB7 may occur via regulation of p53 interactions with Bcl-2 family proteins, which may promote the expression of BNIP3 and the activation of anti-apoptotic Bcl-2. Furthermore, 3-MA pretreatment inhibited mitophagy, aggravated apoptotic cell death and reversed improvement on neurological deficits induced by EA (GV20-GB7) treatment. These findings contribute to knowledge concerning mitophagy induction in $\mathrm{ICH}$ and suggest that regulating the balance between mitophagy and apoptosis may be one mechanism by which EA (GV20-GB7) improves recovery in $\mathrm{ICH}$.

\section{Acknowledgements}

The authors would like to thank to Mr. Haorui Wang of Royal College of Art for providing technical assistance and useful discussion.

\section{Funding}

The present study was supported by the National Natural Science Foundation of China (grant no. 81473764,81273824), the Key Program of Natural Science Foundation of Heilongjiang Province of China (grant no. ZD 201204), the Doctoral Fund of Ministry of Education of China (grant no. 20102327110003) and the Science Foundation for Young Scholars of Zhongshan Hospital, Fudan University (grant no. 2020ZSQN65).

\section{Availability of data and materials}

The datasets used and/or analyzed during the current study are available from the corresponding author on reasonable request.

\section{Authors' contributions}

RG performed the experiments and statistical analysis, collected and analyzed data and wrote the manuscript. ZL performed the experiments, proofread the manuscript and constructed figures. XL, PL and SD collected samples and data, and performed the experiments, including rat $\mathrm{ICH}$ surgery. WZ contributed to the conception and design of the study and obtained funding. XY, XD, XL, HL, QC and WT participated in conceptualization and design of the study and critically revised the manuscript for important intellectual content. RG and WZ confirm the authenticity of all the raw data. All authors read and approved the final manuscript.

\section{Ethics approval and consent to participate}

The present study was approved by the Ethics Committee of the Heilongjiang University of Chinese Medicine (approved no. 2017061001).

\section{Patient consent for publication}

Not applicable.

\section{Competing interests}

The authors declare they have no competing interests.

\section{References}

1. Caplan LR: Intracerebral haemorrhage. Lancet 339: 656-658, 1992.

2. Broderick J, Connolly S, Feldmann E, Hanley D, Kase C, Krieger D, Mayberg M, Morgenstern L, Ogilvy CS, Vespa P, et al: Guidelines for the management of spontaneous intracerebral hemorrhage in adults: 2007 update: A guideline from the American Heart Association/American Stroke Association Stroke Council, High Blood Pressure Research Council, and the Quality of Care and Outcomes in Research Interdisciplinary Working Group. Stroke 38: 2001-2023, 2007.

3. Counsell C, Boonyakarnkul S, Dennis M, Sandercock P, Bamford J, Burn J and Warlow C: Primary intracerebral haemorrhage in the oxfordshire community stroke Project. Cerebrovascular Dis 5: 26-34, 1995.

4. Keep RF, Hua Y and Xi G: Intracerebral haemorrhage: Mechanisms of injury and therapeutic targets. Lancet Neurol 11: 720-731, 2012. 
5. Nehls DG, Mendelow AD, Graham DI, Sinar EJ and Teasdale GM: Experimental intracerebral hemorrhage: Progression of hemodynamic changes after production of a spontaneous mass lesion. Neurosurgery 23: 439-444, 1988.

6. Belur PK, Chang JJ, He S, Emanuel BA and Mack WJ: Emerging experimental therapies for intracerebral hemorrhage: Targeting mechanisms of secondary brain injury. Neurosurg Focus 34: E9 2013.

7. Levine B and Kroemer G: Autophagy in the pathogenesis of disease. Cell 132: 27-42, 2008

8. Niu M, Dai X, Zou W, Yu X, Teng W, Chen Q, Sun X, Yu W, $\mathrm{Ma} \mathrm{H}$ and Liu P: Autophagy, endoplasmic reticulum stress and the unfolded protein response in intracerebral hemorrhage. Transl Neurosci 8: 37-48, 2017.

9. Klionsky DJ and Emr SD: Autophagy as a regulated pathway of cellular degradation. Science 290: 1717-1721, 2000.

10. Wang K and Klionsky DJ: Mitochondria removal by autophagy. Autophagy 7: 297-300, 2014.

11. Goldman SJ, Taylor R, Zhang Y and Jin S: Autophagy and the degradation of mitochondria. Mitochondrion 10: 309-315, 2010.

12. Graef $\mathrm{M}$ and Nunnari J: A role for mitochondria in autophagy regulation. Autophagy 7: 1245-1246, 2011.

13. Wei H, Liu L and Chen Q: Selective removal of mitochondria via mitophagy: Distinct pathways for different mitochondrial stresses. Biochim Biophys Acta 1853: 2784-2790, 2015.

14. Lin C, Chao H, Li Z, Xu X, Liu Y, Hou L, Liu N and Ji J: Melatonin attenuates traumatic brain injury-induced inflammation: A possible role for mitophagy. J Pineal Res 61: 177-186, 2016.

15. Yuan Y, Zheng Y, Zhang X, Chen Y, Wu X, Wu J, Shen Z, Jiang L, Wang L, Yang W, et al: BNIP3L/NIX-mediated mitophagy protects against ischemic brain injury independent of PARK2. Autophagy 13: 1754-1766, 2017.

16. Li J, Lu J, Mi Y, Shi Z, Chen C, Riley J and Zhou C: Voltage-dependent anion channels (VDACs) promote mitophagy to protect neuron from death in an early brain injury following a subarachnoid hemorrhage in rats. Brain Res 1573: 74-83, 2014.

17. Yang Y, Xing D, Zhou F and Chen Q: Mitochondrial autophagy protects against heat shock-induced apoptosis through reducing cytosolic cytochrome c release and downstream caspase-3 activation. Biochem Biophys Res Commun 395: $190-195,2010$

18. Colbert AP, Spaulding K, Larsen A, Ahn AC and Cutro JA Electrodermal activity at acupoints: Literature review and recommendations for reporting clinical trials. J Acupunct and Meridian Stud 4: 5-13, 2011.

19. Zhang S, Wu B, Liu M, Li N, Zeng X, Liu H, Yang O, Han Z, Rao P and Wang D; all Investigators: Acupuncture efficacy on ischemic stroke recovery: Multicenter randomized controlled trial in China. Stroke 46: 1301-1306, 2015.

20. Langevin HM, Schnyer R, MacPherson H, Davis R, Harris RE, Napadow V, Wayne PM, Milley RJ, Lao L, Stener-Victorin E, et al: Manual and electrical needle stimulation in acupuncture research: Pitfalls and challenges of heterogeneity. J Altern Complement Med 21: 113-128, 2015

21. Zhou F, Guo J, Cheng J, Wu G and Xia Y: Electroacupuncture increased cerebral blood flow and reduced ischemic brain injury: Dependence on stimulation intensity and frequency. J Appl Physiol (1985) 111: 1877-1887, 2011

22. Zhu Y, Deng L, Tang H, Gao X, Wang Y, Guo K, Kong J and Yang C: Electroacupuncture improves neurobehavioral function and brain injury in rat model of intracerebral hemorrhage. Brain Res Bull 131: 123-132, 2017.

23. Li HQ, Li Y, Chen ZX, Zhang XG, Zheng XW, Yang WT, Chen S and Zheng GQ: Electroacupuncture exerts neuroprotection through caveolin-1 mediated molecular pathway in intracerebral hemorrhage of rats. Neural Plast 2016: 7308261, 2016.

24. Ting Z, Jianbin Z and Luqi H: Protective effect of electroacupuncture on neurons autophagy in perfusion period of cerebral ischemia. Neurosci Lett 661: 41-45, 2017.

25. Wu Z, Zou Z, Zou R, Zhou X and Cui S: Electroacupuncture pretreatment induces tolerance against cerebral ischemia/reperfusion injury through inhibition of the autophagy pathway. Mol Med Rep 11: 4438-4446, 2015.

26. Liu W, Shang G, Yang S, Huang J, Xue X, Lin Y, Zheng Y, Wang X, Wang L, Lin R, et al: Electroacupuncture protects against ischemic stroke by reducing autophagosome formation and inhibiting autophagy through the mTORC1-ULK1 complex-Beclin1 pathway. Int J Mol Med 37: 309-318, 2016.
27. Wu ZQ, Cui SY, Zhu L and Zou ZQ: Study on the mechanism of mTOR-Mediated autophagy during electroacupuncture pretreatment against cerebral ischemic injury. Evid Based Complement Alternat Med 2016: 9121597, 2016.

28. Zou W, Chen QX, Sun XW, Chi QB, Kuang HY, Yu XP and Dai XH: Acupuncture inhibits Notch1 and Hes1 protein expression in the basal ganglia of rats with cerebral hemorrhage. Neural Regen Res 10: 457-462, 2015.

29. Liu H, Sun X, Zou W, Leng M, Zhang B, Kang X, He T and Wang H: Scalp acupuncture attenuates neurological deficits in a rat model of hemorrhagic stroke. Complement Ther Med 32: 85-90, 2017

30. Health N: Guide for the care and use of laboratory animals. NIH contract No. No1-RR-2-2135: 11-28, 1985.

31. Guan R, Zou W, Dai X, Yu X, Liu H, Chen Q and Teng W: Mitophagy, a potential therapeutic target for stroke. J Biomed Sci 25: 87, 2018.

32. Li Q, Zhang T, Wang J, Zhang Z, Zhai Y, Yang GY and Sun X: Rapamycin attenuates mitochondrial dysfunction via activation of mitophagy in experimental ischemic stroke. Biochem Biophys Res Commun 444: 182-188, 2014.

33. Di Y, He YL, Zhao T, Huang X, Wu KW, Liu SH, Zhao YQ, Fan M, Wu LY and Zhu LL: Methylene blue reduces acute cerebral ischemic injury via the induction of mitophagy. Mol Med 21: 420-429, 2015

34. Jing CH, Wang L, Liu PP, Wu C, Ruan D and Chen G: Autophagy activation is associated with neuroprotection against apoptosis via a mitochondrial pathway in a rat model of subarachnoid hemorrhage. Neuroscience 213: 144-153, 2012.

35. Liu XY, Dai XH, Zou W, Yu XP, Teng W, Wang Y, Yu WW Ma HH, Chen QX, Liu P, et al: Acupuncture through Baihui (DU20) to Qubin (GB7) mitigates neurological impairment after intracerebral hemorrhage. Neural Regen Res 13: 1425-1432, 2018.

36. Zhang B, Dai XH, Yu XP,Zou W, Teng W, Sun XW, Yu WW, Liu H, Wang H, Sun MJ and Li M: Baihui (DU20)-penetrating-Qubin (GB7) acupuncture inhibits apoptosis in the perihemorrhagic penumbra. Neural Regen Res 13: 1602-1608, 2018

37. Wang $\mathrm{C}, \mathrm{Hu} \mathrm{Q}$ and Shen HM: Pharmacological inhibitors of autophagy as novel cancer therapeutic agents. Pharmacol Res 105: 164-175, 2016

38. Hua XB: On animal acupoints. J Tradit Chi Med 7: 301-304, 1987.

39. Jittiwat J: Laser Acupuncture at GV20 improves brain damage and oxidative stress in animal model of focal ischemic stroke. J Acupunct Meridian Stud 10: 324-330, 2017.

40. Yin CS, Jeong HS, Park HJ, Baik Y, Yoon MH, Choi CB and Koh HG: A proposed transpositional acupoint system in a mouse and rat model. Res Vet Sci 84: 159-165, 2008.

41. Han HJ, Park SJ, Soh KS, Myoung HS, Lee KJ, Ogay V and Lee YH: Electrical characterization of proposed transpositional acupoints on the urinary bladder meridian in a rat model. Evid Based Complement Alternat Med 2011: 295475, 2011.

42. Chen J, Sanberg PR, Li Y, Wang L, Lu M, Willing AE, Sanchez-Ramos $\mathbf{J}$ and Chopp M: Intravenous administration of human umbilical cord blood reduces behavioral deficits after stroke in rats. Stroke 32: 2682-2688, 2001

43. Choe SC, Hamacher-Brady A and Brady NR: Autophagy capacity and sub-mitochondrial heterogeneity shape Bnip3-induced mitophagy regulation of apoptosis. Cell Commun Signal 13: 37, 2015.

44. Gross A, McDonnell JM and Korsmeyer SJ: BCL-2 family members and the mitochondria in apoptosis. Genes Dev 13: 1899-1911, 1999

45. Chen B, Wang G, Li W, Liu W, Lin R, Tao J, Jiang M, Chen L and Wang Y: Memantine attenuates cell apoptosis by suppressing the calpain-caspase-3 pathway in an experimental model of ischemic stroke. Exp Cell Res 351: 163-172, 2017.

46. Wagner DC, Riegelsberger UM, Michalk S, Hartig W, Kranz A and Boltze J: Cleaved caspase-3 expression after experimental stroke exhibits different phenotypes and is predominantly non-apoptotic. Brain Res 1381: 237-242, 2011.

47. Yan J, Yun H, Yang Y, Jing B, Feng C and Song-bin F: Upregulation of BNIP3 promotes apoptosis of lung cancer cells that were induced by p53. Biochem Biophys Res Commun 346: 501-507, 2006

48. Tasdemir E, Chiara Maiuri M, Morselli E, Criollo A, D'Amelio M, Djavaheri-Mergny M, Cecconi F, Tavernarakis N and Kroemer G: A dual role of p53 in the control of autophagy. Autophagy 4: 810-814, 2008. 
49. Sims NR and Muyderman H: Mitochondria, oxidative metabolism and cell death in stroke. Biochim Biophys Acta 1802: 80-91, 2010.

50. Kubli DA and Gustafsson AB: Mitochondria and mitophagy: The yin and yang of cell death control. Circ Res 111: 1208-1221, 2012 .

51. Zhang X, Yan H, Yuan Y, Gao J, Shen Z, Cheng Y, Shen Y, Wang RR, Wang X, Hu WW, et al: Cerebral ischemia-reperfusion-induced autophagy protects against neuronal injury by mitochondrial clearance. Autophagy 9: 1321-1333, 2013.

52. Shen Z, Zheng Y, Wu J, Chen Y, Wu X, Zhou Y, Yuan Y, Lu S, Jiang L, Qin Z, et al: PARK2-dependent mitophagy induced by acidic postconditioning protects against focal cerebral ischemia and extends the reperfusion window. Autophagy 13: 473-485, 2017.

53. Baek SH, Noh AR, Kim KA, Akram M, Shin YJ, Kim ES, Yu SW, Majid A and Bae ON: Modulation of mitochondrial function and autophagy mediates carnosine neuroprotection against ischemic brain damage. Stroke 45: 2438-2443, 2014.

54. Shi RY, Zhu SH, Li V, Gibson SB, Xu XS and Kong JM: BNIP3 interacting with LC3 triggers excessive mitophagy in delayed neuronal death in stroke. CNS Neurosci Ther 20: 1045-1055, 2014.

55. Wu M, Lu G, Lao YZ, Zhang H, Zheng D, Zheng ZQ, Yi J, Xiang Q, Wang LM, Tan HS, et al: Garciesculenxanthone B induces PINK1-Parkin-mediated mitophagy and prevents ischemia-reperfusion brain injury in mice. Acta Pharmacol Sin 42: 199-208, 2021.

56. Yin ZL, Meng ZX, Ge S, Zhang MJ and Huang LH: Clinical observation of dynamic scalp acupuncture combined with task-oriented mirror therapy for upper limbs function impairment in patients with hemiplegia after ischemic stroke. Zhongguo Zhen Jiu 40: 918-922, 2020 (In Chinese).

57. Wang WW, Xie CL, Lu L and Zheng GQ: A systematic review and meta-analysis of Baihui (GV20)-based scalp acupuncture in experimental ischemic stroke. Sci Rep 4: 3981, 2014.

58. Zheng GQ, Zhao ZM, Wang Y, Gu Y, Li Y, Chen XM, Fu SP and Shen J: Meta-analysis of scalp acupuncture for acute hypertensive intracerebral hemorrhage. J Altern Complement Med 17: 293-299, 2011

59. Lang Y, Cui FY, Li KS, Tan ZJ and Zou YH: Imaging observation of scalp acupuncture on brain gray matter injury in stroke patients with cerebral infarction. Zhongguo Zhong Xi Yi Jie He Za Zhi 36: 294-299, 2016 (In Chinese).

60. Wang HQ, Bao CL, Jiao ZH and Dong GR: Efficacy and safety of penetration acupuncture on head for acute intracerebral hemorrhage: A randomized controlled study. Medicine (Baltimore) 95: e5562, 2016.

61. Sun ST, Li SR, Zhu YZ, Chen SL, Wan GZ, Sun YZ, Hou GW and Yu ZH: Clinical study on 500 cases of cerebro-vascular hemiplegia treated by acupuncture through baihui to qubin. J Tradit Chin Med 5: 167-170, 1985.

62. Wu Y, Wang X, Guo H, Zhang B, Zhang XB, Shi ZJ and Yu L: Synthesis and screening of 3-MA derivatives for autophagy inhibitors. Autophagy 9: 595-603, 2013.

63. Shao A, Wang Z, Wu H, Dong X, Li Y, Tu S, Tang J, Zhao M, Zhang $\mathrm{J}$ and Hong Y: Enhancement of autophagy by histone deacetylase inhibitor trichostatin a ameliorates neuronal apoptosis after subarachnoid hemorrhage in rats. Mol Neurobiol 53: 18-27, 2016.

64. Xia DY, Li W, Qian HR, Yao S, Liu JG and Qi XK: Ischemia preconditioning is neuroprotective in a rat cerebral ischemic injury model through autophagy activation and apoptosis inhibition. Braz J Med Biol Res 46: 580-588, 2013.

65. Ma H, Chen H, Dong A, Wang Y, Bian Y and Xie K: Hydrogen-rich saline attenuates hyperalgesia and reduces cytokines in rats with post-herpetic neuralgia via activating autophagy. Xi Bao Yu Fen Zi Mian Yi Xue Za Zhi 33: 155-158, 2017 (In Chinese)

66. Tang Y, Cai QH, Wang YJ, Fan SH, Zhang ZF, Xiao MQ, Zhu JY, Wu DM, Lu J and Zheng YL: Protective effect of autophagy on endoplasmic reticulum stress induced apoptosis of alveolar epithelial cells in rat models of COPD. Biosci Rep 37: BSR20170803, 2017.

67. Wu Q, Gao C, Wang H, Zhang X, Li Q, Gu Z, Shi X, Cui Y, Wang T, Chen X, et al: Mdivi-1 alleviates blood-brain barrier disruption and cell death in experimental traumatic brain injury by mitigating autophagy dysfunction and mitophagy activation. Int J Biochem Cell Biol 94: 44-55, 2018.
68. Guo Z, Cao G, Yang H, Zhou H, Li L, Cao Z, Yu B and Kou J: A combination of four active compounds alleviates cerebral ischemia-reperfusion injury in correlation with inhibition of autophagy and modulation of AMPK/mTOR and JNK pathways. J Neurosci Res 92: 1295-1306, 2014

69. Chen X, Wang L, Deng Y, Li X, Li G, Zhou J, Cheng D, Yang Y, Yang Q, Chen G and Wang G: Inhibition of autophagy prolongs recipient survival through promoting CD8(+) T cell apoptosis in a rat liver transplantation model. Front Immunol 10: 1356, 2019.

70. Cao S, Shrestha S, Li J, Yu X, Chen J, Yan F, Ying G, Gu C, Wang L and Chen G: Melatonin-mediated mitophagy protects against early brain injury after subarachnoid hemorrhage through inhibition of NLRP3 inflammasome activation. Sci Rep 7: 2417, 2017.

71. Zuo W, Zhang S, Xia CY, Guo XF, He WB and Chen NH: Mitochondria autophagy is induced after hypoxic/ischemic stress in a Drp1 dependent manner: The role of inhibition of Drp1 in ischemic brain damage. Neuropharmacology 86: 103-115, 2014.

72. Hanna RA, Quinsay MN, Orogo AM, Giang K, Rikka S and Gustafsson AB: Microtubule-associated protein 1 light chain 3 (LC3) interacts with Bnip3 protein to selectively remove endoplasmic reticulum and mitochondria via autophagy. J Biol Chem 287: 19094-19104, 2012.

73. Zhang J and Ney PA: Role of BNIP3 and NIX in cell death, autophagy, and mitophagy. Cell Death Differ 16: 939-946, 2009.

74. Tan H, Wu Z, Wang H, Bai B, Li Y, Wang X, Zhai B, Beach TG and Peng J: Refined phosphopeptide enrichment by phosphate additive and the analysis of human brain phosphoproteome. Protemomics 15: 500-507, 2015.

75. Liu L, Sakakibara K, Chen Q and Okamoto K: Receptor-mediated mitophagy in yeast and mammalian systems. Cell Res 24: 787-795, 2014

76. Kissová I, Plamondon L-T, Brisson L, Priault M, Renouf V, Schaeffer J, Camougrand N and Manon S: Evaluation of the roles of apoptosis, autophagy, and mitophagy in the loss of plating efficiency induced by Bax expression in yeast. J Biol Chem 281: 36187-36197, 2006

77. Li XX, Tsoi B, Li YF, Kurihara H and He RR: Cardiolipin and its different properties in mitophagy and apoptosis. J Histochem Cytochem 63: 301-311, 2015.

78. Levine B, Sinha SC and Kroemer G: Bcl-2 family members: Dual regulators of apoptosis and autophagy. Autophagy 4: 600-606, 2008.

79. Ney PA: Mitochondrial autophagy: Origins, significance, and role of BNIP3 and NIX. Biochim Biophys Acta 1853: 2775-2783, 2015.

80. Feng X, Liu X, Zhang W and Xiao W: p53 directly suppresses BNIP3 expression to protect against hypoxia-induced cell death. EMBO J 30: 3397-3415, 2011

81. Hoshino A, Matoba S, Iwai-Kanai E, Nakamura H, Kimata M, Nakaoka M, Katamura M, Okawa Y, Ariyoshi M, Mita Y, et al: p53-TIGAR axis attenuates mitophagy to exacerbate cardiac damage after ischemia. J Mol Cell Cardiol 52: 175-184, 2012.

82. Marino G, Niso-Santano M, Baehrecke EH and Kroemer G: Self-consumption: The interplay of autophagy and apoptosis. Nat Rev Mol Cell Biol 15: 81-94, 2014.

83. Jevtić G, Nikolić T, Mirčić A, Stojković T, Velimirović M, Trajković V, Marković I, Trbovich AM, Radonjić NV and Petronijević ND: Mitochondrial impairment, apoptosis and autophagy in a rat brain as immediate and long-term effects of perinatal phencyclidine treatment-influence of restraint stress. Prog Neuropsychopharmacol Biol Psychiatry 66: 87-96, 2016.

84. Chipuk JE, Kuwana T, Bouchier-Hayes L, Droin NM, Newmeyer DD, Schuler M and Green DR: Direct activation of Bax by 553 mediates mitochondrial membrane permeabilization and apoptosis. Science 303: 1010-1014, 2004

85. Livesey KM, Kang R, Vernon P, Buchser W, Loughran P, Watkins SC, Zhang L, Manfredi JJ, Zeh HJ III, Li L, et al: p53/HMGB1 complexes regulate autophagy and apoptosis. Cancer Res 72: 1996-2005, 2012.

86. Culmsee C and Mattson MP: p53 in neuronal apoptosis. Biochem Biophys Res Commun 331: 761-777, 2005.

87. Morselli E, Tasdemir E, Maiuri MC, Galluzzi L, Kepp O, Criollo A, Vicencio JM, Soussi T and Kroemer G: Mutant p53 protein localized in the cytoplasm inhibits autophagy. Cell Cycle 7: 3056-3061, 2008

88. Chipuk JE and Green DR: p53's believe it or not: Lessons on transcription-independent death. J Clin Immunol 23: 355-361, 2003.

89. Moll UM and Zaika A: Nuclear and mitochondrial apoptotic pathways of p53. FEBS Lett 493: 65-69, 2001 
90. Wang EY, Gang H, Aviv Y, Dhingra R, Margulets V and Kirshenbaum LA: p53 mediates autophagy and cell death by a mechanism contingent on Bnip3. Hypertension 62: 70-77, 2013.

91. Hendgen-Cotta UB, Esfeld S, Rudi K, Miinalainen I, Klare JP and Rassaf T: Cytosolic BNIP3 dimer interacts with mitochondrial BAX forming heterodimers in the mitochondrial outer membrane under basal conditions. Int J Mol Sci 18: 687, 2017.

92. Namas RA, Metukuri MR, Dhupar R, Velosa C, Jefferson BS, Myer E, Constantine GM, Billiar TR, Vodovotz Y and Zamora R: Hypoxia-induced overexpression of BNIP3 is not dependent on hypoxia-inducible factor $1 \alpha$ in mouse hepatocytes. Shock 36 : 196-202, 2011

93. Hanna Akram R: Bnip3 interacts with LC3 to induce selective removal of endoplasmic reticulum and mitochondria via autophagy. UC San Diego Electronic Theses \& Dissertations: 12-33, 2011 https://escholarship.org/content/qt1wq0k372/qt1wq0k372.pdf. Accessed July 1, 2019.
94. Durcan TM and Fon EA: The three 'P's of mitophagy: PARKIN, PINK1, and post-translational modifications. Genes Dev 29: 989-999, 2015.

95. Chen Y and Dorn GW: PINK1-phosphorylated mitofusin 2 is a parkin receptor for culling damaged mitochondria. Science 340 : 471-475, 2013.

96. Kim-Han J, Kopp S, Dugan L and Diringer M: Perihematomal mitochondrial dysfunction after intracerebral Hemorrhage. Stroke 37: 2457-2462, 2006.

This work is licensed under a Creative Commons Attribution-NonCommercial-NoDerivatives 4.0 International (CC BY-NC-ND 4.0) License. 